\title{
A New Approach to the Synthesis of Benzo[b]naphtho[2,3-b]fu- ran-6,11-diones and 2-Benzyl-3-hydroxynaphthalene-1,4-diones
}

\author{
Jose C. Barcia ${ }^{a}$ \\ Jacobo Cruces ${ }^{\mathrm{b}}$ \\ Cristian O. Salas*c \\ Juan C. Estévez \\ Mauricio A. Cuellar \\ Ricardo A. Tapiac \\ Ramón J. Estévez*a \\ a Centro Singular de Investigación en Química Biolóxica e Mate- \\ riales Moleculares and Departamento de Química Orgánica, \\ Universidade de Santiago de Compostela, 15782 Santiago de \\ Compostela, Spain \\ ramon.estevez@usc.es \\ b GalChimia S.A., Cebreiro s/n, 15823 O Pino, A Coruña, Spain \\ c Departamento de Química Orgánica, Facultad de Química, \\ Pontificia Universidad Católica de Chile, 702843 Santiago de Chile, Chile \\ cosalas@uc.cl \\ Received: 11.09.2017 \\ Accepted after revision: 02.11.2017 \\ Published online: 27.11 .2017 \\ DOI: 10.1055/s-0036-1591729; Art ID: so-2017-d0040-op \\ License terms: (c)
}

${ }^{d}$ Facultad de Farmacia, Universidad de Valparaíso, Av. Gran Bretaña No. 1093, Valparaíso 2340000, Chile
Abstract Here we describe modified syntheses of 0 -acetylbenzoic acids and o-acetylphenylacetic acids by Heck palladium-catalysed arylation of $n$-butyl vinyl ether with o-iodobenzoic acids or with o-iodophenylacetic acids, respectively. General syntheses of benzo[b]naphtho[2,3-b]furan-6,11-diones from o-acetylbenzoic acids and 2-benzyl3-hydroxynaphthalene-1,4-diones from 0 -acetylphenylacetic acids are also reported.

Key words fused-ring systems, Heck reaction, quinones, palladium, heterocycles, halides, furans

The synthesis of naphthoquinones is of great significance because of the widespread occurrence of the 1,4naphthoquinone nucleus in numerous natural and synthetic compounds of biological and industrial interest. ${ }^{1-8}$ Specifically, considerable attention has been devoted to 2-hydroxy-1,4-naphthoquinones (I) and 2-hydroxy-3-phenyl1,4-naphthoquinones (II) (Figure 1), on account of their biological properties, their industrial applications, and their potential as intermediates in the synthesis of oxygenated and nitrogenated heterocyclic quinones, including $5 \mathrm{H}$-benzo[b]carbazole-6,11-diones (benzocarbazolequinones) ${ }^{9,10}$ benzo[ $b]$ naphtho[2,3- $b]$ furan-6,11-diones (benzofuronaphthoquinones $)^{11,12}$ and $5 H$-dibenzo[c,g]chromene5,7,12-triones (benzopyronaphtho-quinones). ${ }^{13,14}$ Benzocarbazolequinones (III, Figure 1) became important synthetic targets once their antineoplastic activity was established. ${ }^{15-18}$ On the other hand, a representative example of benzofuronaphthoquinones is compound IV (Figure 1) and a representative example of benzopyronaphthoquinones $\mathbf{V}$
(Figure 1) is the quinonoid anticoccidial antibiotic WS5995-A. ${ }^{19-21}$ The antineoplastic activity displayed by compounds III, IV and $\mathbf{V}$ has been related to the well-known antitumor properties of ellipticine (Figure 1 ). ${ }^{15-18}$ The antineoplastic activity of these compounds has been attributed to their ring systems, which contain an embedded 2phenylnaphthalene-like structure in a planar conformation, which facilitates its intercalation between adjacent pairs of DNA bases, thereby interfering with DNA replication and transcription. ${ }^{22}$ In addition, the quinone moiety present in the ring skeleton explains the cytotoxic properties of these compounds and enhances the strength of intercalative binding to DNA through the formation of charge-transfer interactions with the electron-rich DNA bases. ${ }^{23-25}$<smiles>O=C1C=C(O)C(=O)c2ccccc21</smiles>

I<smiles>O=C1c2ccccc2C(=O)c2c1oc1cc(O)cc(O)c21</smiles>

IV<smiles>O=C1C(O)=C(c2ccccc2)C(=O)c2ccccc21</smiles>

II<smiles>O=C1c2ccccc2C(=O)c2c1[nH]c1ccccc21</smiles>

III

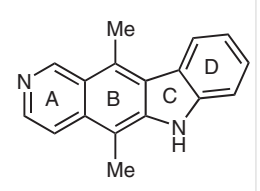

Ellipticine

Figure 1 Representative 2-hydroxy-1,4-naphthoquinones and tetracyclic naphthoquinones related to ellipticine

From a chemical point of view, particular interest has been devoted to 2-hydroxy-3-phenyl-1,4-naphthoquinones (VI, $m=0$, Scheme 1 ), because they proved to be convenient 


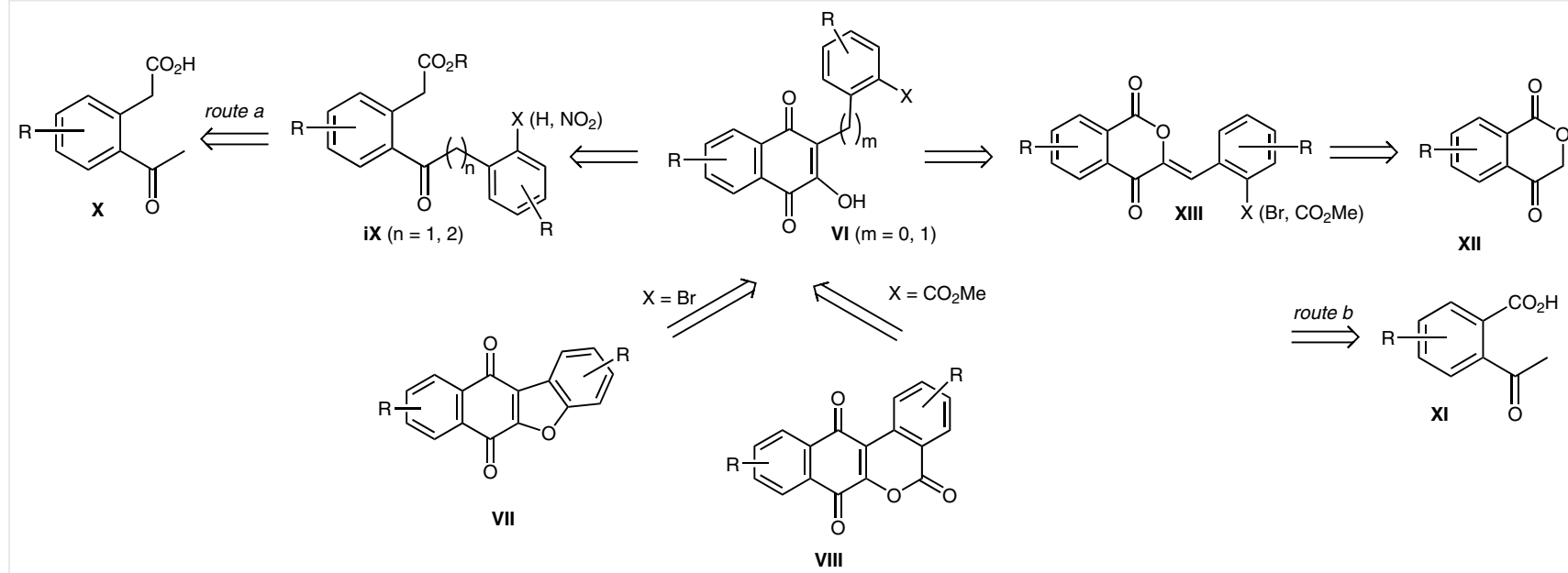

Scheme 1 Retrosynthetic plan towards benzofuronaphthoquinones VII, benzopyronaphthoquinones VIII and 2-hydroxy-3-benzyl-1,4-naphothoquinones $\mathbf{V I}(m=1)$

precursors for the synthesis of naphthoquinone derivatives VII, VIII and IX, and related compounds. ${ }^{15-18}$ Accordingly, a range of methods for the preparation of these powerful scaffolds have been developed, including our previously reported general syntheses from 0 -acetylphenylacetic acids $\mathbf{X I}(\text { route } a)^{26}$ and from $o$-acetylbenzoic acids XII (route $\left.b\right){ }^{27}$ Route $a$ was found to be of limited scope because it only allowed benzocarbazolequinones III to be prepared. These tetracyclic naphthoquinones were alternatively obtained through route $b$, by a sequence involving the condensation of isochroman-1,4-diones XII with p-nitrobenzaldehydes, followed by rearrangement of the resulting 3-benzylideneisochroman-1,4-diones $\mathbf{X I I I}\left(\mathrm{X}=\mathrm{NO}_{2}\right)$ to the corresponding naphthoquinones $(m=0)$ and subsequent generation of the nitrogen ring. ${ }^{28,29}$ These two general approaches to benzocarbazolequinones allowed the limitations of previous syntheses of these targets to be overcome. ${ }^{10,11,30-32}$ As a continuation of this work, herein we report studies on the synthesis of benzofuronaphthoquinones VII and benzopyronaphthoquinones VIII from 0 -acetylbenzoic acids XI, via 3-hydroxy2-phenylnaphthoquinones VI (Scheme 1). The synthesis of 2-benzyl-3-hydroxynaphthalene-1,4-diones (VI, $m=1$ ) and $\boldsymbol{o}$-acetylphenylacetic acids $\mathbf{X}$ is also described.
Our previous, general and straightforward synthesis 0 acetylbenzoic acids involved a Heck coupling reaction between electron-rich $n$-butyl vinyl ether (BVE) and 2-bromobenzoates 1a-b, using the conditions described by Cabri et al. ${ }^{33}$ (Scheme 2). ${ }^{27}$ Firstly, a Heck reaction between BVE and methyl $\boldsymbol{o}$-bromobenzoate (1a) provided the expected $\alpha$-arylation product $2 \mathbf{a}$ only. This regiochemical outcome was attributed to the presence of TIOAc and a chelating phosphine in the reaction medium. The aryl vinyl ether $\mathbf{2 a}$ was immediately reacted with $10 \%$ aqueous $\mathrm{HCl}$ for 1 hour at room temperature, to afford ketoester $3 a$ in $90 \%$ yield. On the other hand, the coupling reaction between the electron-rich methyl 2-bromo-4,5-dimethoxybenzoate dimethoxy derivative 1b and BVE, under the same conditions, gave ketoester $\mathbf{3 b}$ in lower yield, via aryl vinyl ether $\mathbf{2 b}$.

Surprisingly, the same regioselectivity was observed when the coupling reaction of $\mathbf{1} \mathbf{a}$ and $\mathbf{1} \mathbf{b}$ with BVE was performed under classical Heck conditions, which required longer reaction times, but avoided the use of toxic thallium salts and expensive phosphines. The uncommonly high $\alpha$ regioselectivity achieved under these classical conditions may be the result of an interaction between the 0 -carbomethoxy group of aryl halides $\mathbf{1}$ and the palladium complex involved in the Heck coupling.

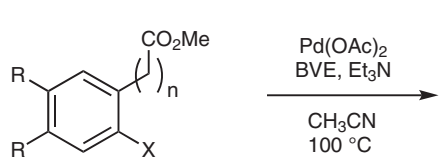

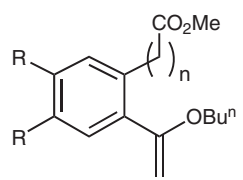

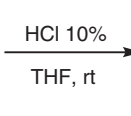

$$
\begin{aligned}
& \text { 2a: } R=H(n=0) \\
& \text { 2b: } R=O M e(n=0) \\
& \text { 2c: } R=H(n=1) \\
& \text { 2d: } R=O M e(n=1)
\end{aligned}
$$<smiles>[R]c1cc(C(C)=O)c(C(C)=O)cc1P</smiles>

3a: $\mathrm{R}=\mathrm{H}(\mathrm{n}=0)$

3b: $R=O M e(n=0)$

3c: $R=H(n=1)$

3d: $R=O M e(n=1)$

Scheme 2 Heck coupling of methyl o-iodobenzoates $\mathbf{1 c , d}$ and methyl o-iodophenylacetates $\mathbf{1 g}, \mathbf{h}$ and with BVE: synthesis of methyl o-acetylbenzoates $\mathbf{3 a}, \mathbf{b}$ and methyl o-acetylphenylacetates $\mathbf{3 c}, \mathbf{d}$ 
Table 1 Formation of $3 a, b$ and $3 c, d$

\begin{tabular}{cclllll}
\hline $\begin{array}{c}\text { Entry } \\
\text { strate }\end{array}$ & Catalyst & Solvent & Time & Product & Yield (\%) \\
\hline 1 & $\mathbf{1 c}$ & $\begin{array}{l}\mathrm{Pd}(\mathrm{OAc})_{2} / \mathrm{Ph}_{3} \mathrm{P} \\
(7.5 \mathrm{~mol} \%)\end{array}$ & $\mathrm{CH}_{3} \mathrm{CN}$ & $4 \mathrm{~h}$ & $\mathbf{3 a}$ & 90 \\
2 & $\mathbf{1 d}$ & $\begin{array}{l}\mathrm{Pd}(\mathrm{OAc})_{2} / \mathrm{Ph}_{3} \mathrm{P} \\
(10 \mathrm{~mol} \%)\end{array}$ & $\mathrm{CH}_{3} \mathrm{CN}$ & $16 \mathrm{~h}$ & $\mathbf{3 b}$ & 84 \\
3 & $\mathbf{1 g}$ & $\begin{array}{l}\mathrm{Pd}(\mathrm{OAc})_{2} / \mathrm{Ph}_{3} \mathrm{P} \\
(2.5 \mathrm{~mol} \%)\end{array}$ & $\mathrm{CH}_{3} \mathrm{CN}$ & $16 \mathrm{~h}$ & $\mathbf{3 c}$ & 68 \\
4 & $\mathbf{1 h}$ & $\begin{array}{l}\mathrm{Pd}(\mathrm{OAc})_{2} / \mathrm{Ph}_{3} \mathrm{P} \\
(2.5 \mathrm{~mol} \%)\end{array}$ & $\mathrm{CH}_{3} \mathrm{CN}$ & 1 week & $\mathbf{3 d}$ & 60 \\
\hline
\end{tabular}

\footnotetext{
${ }^{\text {a }}$ All the experiments were carried out at $100{ }^{\circ} \mathrm{C}$

b See ref. ${ }^{23}$

c See ref.22
}

On the other hand, similar regioselectivities and yields were previously achieved for the coupling of $o$-bromophenylacetates 1e and 1f with BVE, both under the Cabri and classical conditions. $\mathbf{o}$-Acetylphenylacetates $\mathbf{3 c}$ and $\mathbf{3 d}$ were obtained respectively, via the corresponding enol ethers $\mathbf{2 c}$ and $\mathbf{2 d}$.

The similar reaction of BVE with methyl iodobenzoates 1c-d, and with methyl iodophenylacetates $\mathbf{1 g - h}$ was then studied under these classical conditions, in order to assess the influence of the halogen on this Heck coupling reaction. A selective $\alpha$-arylation was again observed, similar reaction times were required, and similar yields were achieved (Table 1 , entries 1,2 and 3,4).

A tentative explanation for the role played by the carbomethoxy group of both types of substrates $(\mathbf{1 a}-\mathbf{d}$ or $\mathbf{1 e}-\mathbf{h})$ in the Heck reaction is depicted in Scheme 3. The insertion of $\operatorname{Pd}(0)$ into the carbon-halogen bond of the starting aryl halide would lead to complex B, via complex A. Dissociation of the halogen atom should give a cationic complex $\mathrm{C}$, with internal association of the methoxycarbonyl group. Removal of a ligand $L$ provided a free position that can be used to link a BVE unit. Next, the 0 -carbomethoxy group could assist the insertion of $\operatorname{Pd}(0)$ through chelation (complex D). ${ }^{34}$ It has been proposed that chelation between the carbonyl group and the palladium atom could play an important role in promoting high regioselectivities. ${ }^{33}$ This may be supported by the fact that the regioselectivity in Heck reactions is dependent on the ionic versus neutral mechanisms pro- posed for this reaction, and that branched alkenes are mainly obtained from electron-rich alkenes, such as BVE, under ionic mechanism conditions. ${ }^{35-38}$ The predominance of electronic over steric effects is responsible for the regioselectivity observed in this reaction.

According to our synthetic plan, methyl o-acetylbenzoate 3a was hydrolysed, by refluxing a solution of this ketoester and $20 \%$ aqueous $\mathrm{H}_{2} \mathrm{SO}_{4}$ under reflux for $2 \mathrm{~h} .{ }^{39}$ This afforded the corresponding benzoic acid lactol $\mathbf{4 a}$ in $95 \%$ yield (Scheme 4 ), which readily provided bromomethyl lactol $\mathbf{5 a}$ in $96 \%$ yield, upon treatment with bromine in acetic acid/toluene. Finally, treatment of $\mathbf{5 a}$ with $\mathrm{NaOAc}$ in ethanol gave isochroman-1,4-dione $\mathbf{6 a}$ in $98 \%$ yield. ${ }^{27,39}$ Ketoester 3b was similarly and efficiently converted into isochroman1,4-dione $\mathbf{6 b}$ via compounds $\mathbf{4 b}$ and $\mathbf{5 b}$.

Reaction of isochroman-1,4-dione 6a with o-bromobenzaldehyde (7a) in ammonium acetate/acetic acid provided the new benzylideneisochroman-1,4-dione $\mathbf{8 a}$ in $81 \%$ yield. Treatment of $\mathbf{8 a}$ with sodium methoxide in methanol gave the known 3-bromophenyl-2-hydroxy-1,4-naphthoquinone 10a in $65 \%$ yield, through rearrangement of intermediate 9a. ${ }^{11} \mathrm{~A}$ similar condensation of isochroman-1,4-dione $\mathbf{6 b}$ with 2-bromo-4,5-dimethoxybenzaldehyde (7b), resulted in the formation of benzylideneisochroman-1,4dione $\mathbf{8 b}$, which rearranged readily to the expected 3-bromophenyl-2-hydroxy-1,4-naphthoquinone 10b. As quinones 10a and 10b were previously converted into benzofuronaphthoquinones 11a and 11b, respectively, $30,31,40$ the present approach constitutes a novel, general synthesis of these tetracyclic quinones, that overcomes limitations of our previous routes.

In an attempt to apply this synthetic strategy to the preparation of $5 \mathrm{H}$-dibenzo[c,g]chromene-5,7,12-triones, when isochroman-1,4-dione $\mathbf{6 a}$ was reacted with 0 -methoxycarbonylbenzaldehyde, under the same conditions as for 8a, the expected benzylideneisochroman-1,4-dione $\mathbf{1 1}$ was obtained in $75 \%$ yield (Scheme 5). However, when this compound was reacted with sodium methoxide in methanol, the resulting compound was not the desired 3-methoxycarbonyl-2-hydroxynaphthoquinone $\mathbf{1 5}$ that should result from intermediate $\mathbf{1 2}$. Compound $\mathbf{1 4}$ was obtained in $70 \%$ yield. Probably, the favoured process is now the lactonisation of enol $\mathbf{1 3}$ of intermediate $\mathbf{1 2}$.

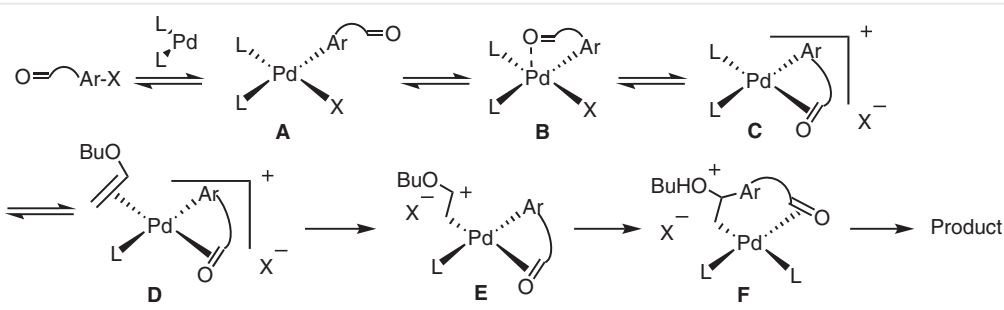

Scheme 3 Mechanistic explanation for the regiochemistry observed in the Heck coupling reaction of methyl o-halophenylacetates and methyl o-halobenzoates with BVE 


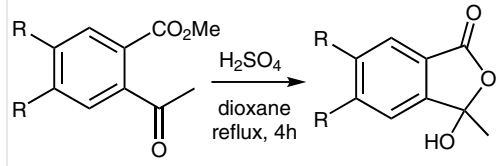

3a: $\mathrm{R}=\mathrm{H}$ 3b: $R=O M e$ 4a: $\mathrm{R}=\mathrm{H}(95 \%)$ 4b: $\mathrm{R}=\mathrm{OMe}(98 \%)$

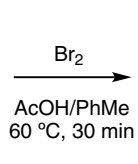
$60^{\circ} \mathrm{C}, 30 \mathrm{~min}$

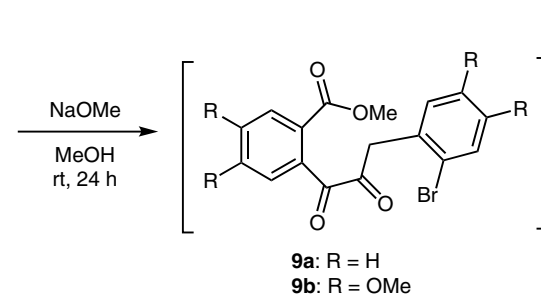<smiles>[R]c1cc2c(cc1P)C(=O)C(c1cc(P)c(P)cc1Br)C(O)C2=O</smiles><smiles>[2H]c1cc2c(cc1[2H])C(O)(CBr)OC2=O</smiles>

5a: $\mathrm{R}=\mathrm{H}(95 \%)$ $5 a: R=H(95 \%)$
$5 b: R=O M e(98 \%)$

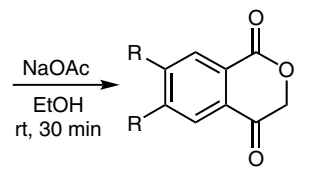

6a: $\mathrm{R}=\mathrm{H}(95 \%)$ 6b: $R=$ OMe $(98 \%)$<smiles>[2H]c1cc(Br)c(/C=C2\OC(=O)c3cc([2H])c([2H])cc3C2=O)cc1[2H]</smiles>

8a: $\mathrm{R}=\mathrm{H}(81 \%)$ 8b: $\mathrm{R}=\mathrm{OMe}(85 \%)$

Scheme 4 Synthesis of benzo[b]naphtho[2,3-b]furanquinones $\mathbf{1 1 a , b}$ from methyl 2-acetylbenzoates via isochroman-1,4-diones $\mathbf{6 a , b}$

On the other hand, it is interesting to note that 3-alkyl2-hydroxy-1,4-naphthoquinones have received considerably less attention than the corresponding 3-phenyl 2-hydroxy-1,4-naphthoquinones. Its most significant component is lapachol, a natural occurring phenolic compound isolated from the bark of the lapacho tree, which possess antitumor and antiparasitic properties. ${ }^{41-44} \mathrm{~A}$ family of compounds structurally related to lapachol are 3-benzyl-2hydroxy-1,4-naphthoquinones, which were previously prepared by alkylation of 2-hydroxy-1,4-naphthoquinones with alkyl halides or by condensation with aldehydes. Thus, alkylation of lawsone with benzyl chlorides, under basic conditions, provided the 3-benzyl-2-hydroxy-1,4-naphthoquinones with a range of $38-43 \%$ yield. ${ }^{42,45}$ Thus 3-benzyl- 2-hydroxy-1,4-naphthoquinones were alternatively obtained by condensation of lawsone with benzaldehydes, in a range of $75-85 \%$ yield. ${ }^{46,47}$

As an additional contribution to this field, we report here the synthesis of 3-benzyl-2-hydroxy-1,4-naphthoquinones 20a-d from 0 -acetylphenylacetic acids $\mathbf{1 6 a}, \mathbf{b}$, which were obtained by hydrolysis of the respective methyl 0 acetylphenylacetates 3c,d (Scheme 6). Thus, condensation of 0 -acetylphenylacetic acid (16a) with benzaldehyde (7c) provided the corresponding $\alpha, \beta$-unsaturated derivative 17a (50\%), which, upon catalytic hydrogenation, gave $o$-phenylpropylphenylacetic acid 18a in high yield (93\%). Subsequent treatment of this ketoacid with $t$ - $\mathrm{BuOK}$ in $t-\mathrm{BuOH}$, resulted in the unreported benzylnaphthoquinone 20a in 57\% yield. Reaction of 17a with 3,4-dimethoxybenzaldehyde (7d) provided the unknown benzylnaphthoquinone 20b in $72 \%$

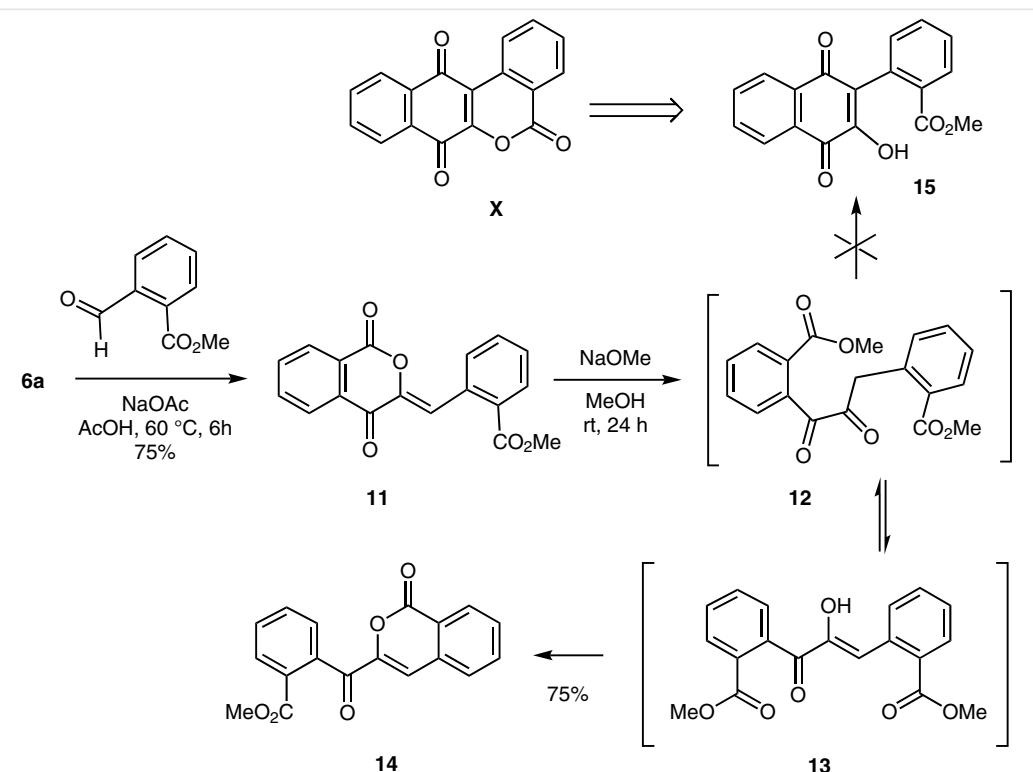

Scheme 5 Unsuccessful approach to the synthesis of $5 \mathrm{H}$-dibenzo[c,g]chromene-5,7,12-triones $\mathbf{X}$ 
yield, via compounds $\mathbf{1 7 b}$ and $\mathbf{1 8 b}$. Reaction of $\boldsymbol{o}$-acetylphenylacetic acid 16b with benzaldehyde gave the known 3benzylnaphthoquinone 20c, via compounds 17c and 18c. Finally, when $\mathbf{1 6 b}$ reacted with 3,4-dimethoxibenzaldehyde, the known benzylnaphthoquinone 20d was obtained in $39 \%$ yield, via compounds 17d and 18d. This new synthesis of 3-benzyl-2-hydroxy-1,4-naphthoquinones proved to be more efficient than previously reported approaches. ${ }^{42,45}$

As a whole, we have revisited our general and efficient method for the preparation of methyl 0 -acetylbenzoates and methyl 0 -acetylphenylacetates. A slight modification consisting of the replacement of the starting 0 -bromobenzoic acid esters and the $\boldsymbol{o}$-bromophenylacetic acid esters by the corresponding aryl iodides, allow these ketoacids to be obtained in similar yields and stereoselectivities.

In addition, a new synthetic application of the 0 -acetylbenzoic acid derived isochroman-1,4-diones, involving transformation into benzo[b]naphtho[2,3-b]furan-6,11-diones was developed. This new, general synthesis of these targets allows easy and efficient access to a variety of antitumor quinones, for chemical and biological studies.

The practically unexplored $\boldsymbol{o}$-phenylpropionylphenylacetic acids are promising scaffolds for the development of a range of further synthetic applications. Interestingly, the 2-benzyl-1,4-naphthoquinone nucleus is embedded in the tetracyclic structures of benzo[ $b$ ]acridine-6,11,12(5H)-triones $^{48}$ and $11 H$-benzo[ $\left.b\right]$ naphtho[2,3-e]pyran-6,11,12-triones. ${ }^{49}$ This structural relationship opens an opportunity for a new synthetic approach to these targets, and provides access to libraries of both practically unexplored kind of compounds, for chemical and biological studies.

Work is in progress aimed at the exploration of these promising chemical goals.

Melting points were determined with a Kofler Thermogerate apparatus and are uncorrected. Infrared spectra were recorded with a JASCO FT/IR-400 spectrophotometer. Nuclear magnetic resonance spectra were recorded, unless otherwise specified, with a Bruker WM-250 apparatus using $\mathrm{CDCl}_{3}$ solutions containing tetramethylsilane (TMS) as internal standard. ${ }^{1} \mathrm{H}$ NMR splitting patterns are designated as singlet (s), doublet (d), triplet (t), quartet (q) or quintuplet (p). All firstorder splitting patterns were assigned based on the appearance of the multiplet. Splitting patterns that could not be easily interpreted are designated as multiplet ( $\mathrm{m}$ ) or broad (br). Mass spectra were obtained with a HP 5988A mass spectrometer. Elemental analyses were performed with an EA 1108 CHNS Fisons instrument. Thin-layer chromatography (TLC) was performed using Merck GF-254 type 60 silica gel and dichloromethane/methanol or EtOAc/hexane mixtures as eluents; the TLC spots were visualised with ultraviolet light or iodine vapour. Column chromatography was carried out using Merck type 9385 silica gel. Solutions of extracts in organic solvents were dried with anhydrous sodium sulphate.

\section{Methyl 2-Acetylbenzoates and Methyl 2-(2-Acetylphenyl)acetates 3; General Procedure}

In a sealed tube fitted with Teflon screw cap, solutions of 1c, 1d, $1 \mathrm{~g}$ and $\mathbf{1 h}(1.2 \mathrm{mmol}), \mathrm{BVE}(0.09 \mathrm{mmol}), \mathrm{Pd}(\mathrm{OAc})_{2}(7.5 \%), \mathrm{PPh}_{3}(0.18$ $\mathrm{mmol})$ and $\mathrm{Et}_{3} \mathrm{~N}(0.90 \mathrm{~mL})$ in anhydrous and deoxygenated $\mathrm{CH}_{3} \mathrm{CN}$ $(2.5 \mathrm{~mL})$, were heated to $100{ }^{\circ} \mathrm{C}$ for $4 \mathrm{~h}$. The respective reaction mixture was filtered through Celite, washed with $\mathrm{CH}_{2} \mathrm{Cl}_{2}$, and the filtrates

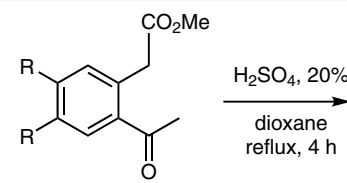

3c: $\mathrm{R}=\mathrm{H}$ 3d: $\mathrm{R}=\mathrm{OMe}$<smiles>[2H]c1cc(CC(=O)O)c(C(C)=O)cc1P</smiles>

16a: $\mathrm{R}=\mathrm{H}(92 \%)$ 16b: $R=O M e(86 \%)$

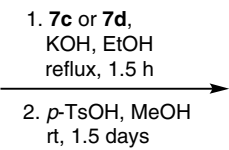

rt, 1.5 days

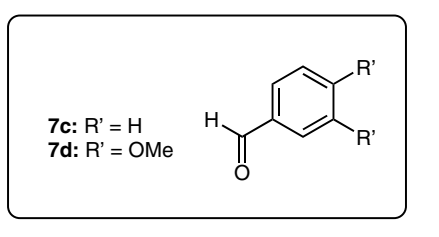<smiles>[2H]c1cc(CC(C)=O)c(C(=O)/C=C/c2ccc(P)c(P)c2)cc1P</smiles>

17a: $R=R^{\prime}=H(50 \%)$

17b: $\mathrm{R}=\mathrm{H}, \mathrm{R}^{\prime}=\mathrm{OMe}(95 \%)$ 17c: $\mathrm{R}=\mathrm{OMe}, \mathrm{R}^{\prime}=\mathrm{H}(87 \%)$ 17d: $R=R^{\prime}=$ OMe (not isolated)<smiles>[2H]c1cc2c(cc1P)C(=O)C(Cc1ccc(F)c(F)c1)=C(O)C2=O</smiles>

20a: $R=R^{\prime}=H^{\prime}(57 \%)$ 20b: $R=H, R^{\prime}=$ OMe $(72 \%)$ 20c: $R=O M e, R^{\prime}=H(54 \%)$ 20d: $R=R^{\prime}=\operatorname{OMe}(39 \%)$

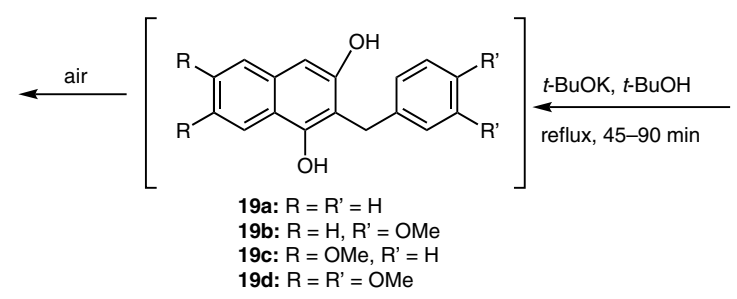

18a: $R=R^{\prime}=H^{\prime}(93 \%)$ 18b: $\mathrm{R}=\mathrm{H}, \mathrm{R}^{\prime}=\mathrm{OMe}(87 \%)$ 18c: $R=O M e, R^{\prime}=H(94 \%)$ 18d: $R=R^{\prime}=$ OMe $(58 \%)$

Scheme 6 Synthesis of 3-benzyl-2-hydroxy-1,4-naphthoquinones 20a-d from o-acetylphenylacetic acids 16a,b 
were washed with distilled water $(25 \mathrm{~mL})$. The organic phases were dried with anhydrous $\mathrm{Na}_{2} \mathrm{SO}_{4}$ and concentrated to dryness. The residues were dissolved in a mixture of $10 \% \mathrm{THF} / \mathrm{HCl}(40 \mathrm{~mL}, 1: 1)$ and the solution were stirred for $2 \mathrm{~h}$ at r.t., the solvent was evaporated and the residues were extracted with $\mathrm{CH}_{2} \mathrm{Cl}_{2}(3 \times 40 \mathrm{~mL})$. The combined organic layers were washed with $10 \%$ aqueous $\mathrm{NaHCO}_{3}$, dried over anhydrous $\mathrm{Na}_{2} \mathrm{SO}_{4}$, and the solvent evaporated off. The residues were purified by column chromatography (EtOAc/hexane, 2:3), to give 3ad.

\section{Methyl 2-Acetylbenzoate (3a) $)^{50}$}

Yield: $182 \mathrm{mg}$ (90\%); colourless oil.

IR: $1710(\mathrm{C}=0) \mathrm{cm}^{-1}$.

${ }^{1} \mathrm{H}$ NMR $\left(\mathrm{CDCl}_{3}\right): \delta=2.53\left(\mathrm{~s}, 3 \mathrm{H}, \mathrm{CH}_{3}\right), 3.89\left(\mathrm{~s}, 3 \mathrm{H}, \mathrm{OCH}_{3}\right), 7.32-7.67$ $(\mathrm{m}, 3 \mathrm{H}, 3 \times \mathrm{Ar}-\mathrm{H}), 7.77-7.92(\mathrm{~m}, 1 \mathrm{H}, \mathrm{Ar}-\mathrm{H})$.

${ }^{13} \mathrm{C} \mathrm{NMR}\left(\mathrm{CDCl}_{3}\right): \delta=29.7\left(\mathrm{CH}_{3}\right), 52.4\left(\mathrm{OCH}_{3}\right), 126.4(\mathrm{CH}), 128.8(\mathrm{C})$, $129.6(\mathrm{CH}), 130.0(\mathrm{CH}), 132.0(\mathrm{CH}), 142.6(\mathrm{C}), 167.4(\mathrm{C}=\mathrm{O}), 202.8$ $(\mathrm{C}=0)$.

MS: $m / z(\%)=178(100)\left[\mathrm{M}^{+}\right]$.

\section{Methyl 2-Acetyl-4,5-dimethoxybenzoate (3b)}

Yield: $186 \mathrm{mg}$ (84\%); white solid; mp $118-120^{\circ} \mathrm{C}$ (EtOAc).

\section{Methyl 2-(2-Acetylphenyl)acetate (3c)}

Yield: $164 \mathrm{mg}$ (68\%); colourless oil.

\section{Methyl 2-(2-Acetyl-4,5-dimethoxyphenyl)acetate (3d)}

Yield: $128 \mathrm{mg}$ (60\%); white solid; $\mathrm{mp} 64-67{ }^{\circ} \mathrm{C}\left(\mathrm{Et}_{2} \mathrm{O}\right)$.

IR (NaCl): $1703(\mathrm{C}=0), 1600(\mathrm{C}=0) \mathrm{cm}^{-1}$.

${ }^{1} \mathrm{H} \mathrm{NMR}\left(\mathrm{CDCl}_{3}\right): \delta=2.58\left(\mathrm{~s}, 3 \mathrm{H}, \mathrm{CH}_{3}\right), 3.70\left(\mathrm{~s}, 3 \mathrm{H}, \mathrm{OCH}_{3}\right), 3.89(\mathrm{~s}, 2 \mathrm{H}$, $\left.\mathrm{CH}_{2}\right) ; 3.93\left(\mathrm{~s}, 6 \mathrm{H}, 2 \times \mathrm{CH}_{3}\right), 6.72(\mathrm{~s}, 1 \mathrm{H}, \mathrm{Ar}-\mathrm{H}), 7.33(\mathrm{~s}, 1 \mathrm{H}, \mathrm{Ar}-\mathrm{H})$.

${ }^{13} \mathrm{C} \mathrm{NMR}\left(\mathrm{CDCl}_{3}\right): \delta=28.5\left(\mathrm{CH}_{3}\right), 40.2\left(\mathrm{CH}_{3}\right), 51.8\left(\mathrm{OCH}_{3}\right), 56.0\left(\mathrm{OCH}_{3}\right)$, $56.2\left(\mathrm{OCH}_{3}\right), 113.9(\mathrm{CH}), 115.5(\mathrm{CH}), 129.2(\mathrm{C}), 129.4(\mathrm{C}), 147.5(\mathrm{Ar}-$ $\left.\mathrm{OCH}_{3}\right), 152.0\left(\mathrm{Ar}^{-} \mathrm{OCH}_{3}\right), 172.2(\mathrm{C}=\mathrm{O}), 199.1(\mathrm{C}=\mathrm{O})$.

MS: $m / z(\%)=239(21)\left[\mathrm{M}^{+}\right], 223(100)$.

Anal. Calcd for $\mathrm{C}_{13} \mathrm{H}_{16} \mathrm{O}_{5}$ : C, 61.90; $\mathrm{H}, 6.39$. Found: $\mathrm{C}, 61.08 ; \mathrm{H}, 6.19$.

\section{3-Hydroxy-3-methyl-3H-isobenzofuran-1-ones 4; General Proce- dure}

$20 \%$ Aqueous $\mathrm{H}_{2} \mathrm{SO}_{4}(10 \mathrm{~mL})$ was added to solutions of $\mathbf{3 a}$ and $\mathbf{3 b}(5.62$ $\mathrm{mmol})$ in dioxane $(18 \mathrm{~mL})$ and the reaction mixtures were heated at reflux for $2 \mathrm{~h}$. The mixtures were allowed to cool to r.t., poured into water $(20 \mathrm{~mL})$ and extracted with $\mathrm{CH}_{2} \mathrm{Cl}_{2}(5 \times 20 \mathrm{~mL})$. The combined organic layers were washed with water $(2 \times 20 \mathrm{~mL})$, dried with anhydrous $\mathrm{Na}_{2} \mathrm{SO}_{4}$ and concentrated to dryness, to give $\mathbf{4 a}$ and $\mathbf{4 b}$, respectively.

\section{3-Hydroxy-3-methyl-3H-isobenzofuran-1-ones (4a)}

Yield: $0.88 \mathrm{~g}$ (95\%); white solid; $\mathrm{mp} 116-118{ }^{\circ} \mathrm{C}\left(\mathrm{CHCl}_{3}\right)$.

IR ( $\mathrm{NaCl}): 3267(\mathrm{OH}), 1726(\mathrm{C}=\mathrm{O}) \mathrm{cm}^{-1}$.

${ }^{1} \mathrm{H}$ NMR $\left(\mathrm{CDCl}_{3}\right): \delta=1.95\left(\mathrm{~s}, 3 \mathrm{H}, \mathrm{CH}_{3}\right), 5.71$ (br s, $\left.1 \mathrm{H}, \mathrm{OH}\right), 7.47-7.60$ (m, $2 \mathrm{H}, 2 \times \mathrm{Ar}-\mathrm{H}), 7.63-7.82(\mathrm{~m}, 2 \mathrm{H}, 2 \times \mathrm{Ar}-\mathrm{H})$.

${ }^{13} \mathrm{C}$ NMR $\left(\mathrm{CD}_{3} \mathrm{OD}\right): \delta=26.4\left(\mathrm{CH}_{3}\right), 108.1(\mathrm{C}), 123.5(\mathrm{CH}), 125.9(\mathrm{CH})$, 127.6 (C), 131.4 (CH), 135.8 (CH), 151.9 (C), 170.4 (C=O).

MS: $m / z(\%)=165(67)\left[\mathrm{M}+\mathrm{H}^{+}\right], 147(100)$.

Anal. Calcd for $\mathrm{C}_{9} \mathrm{H}_{8} \mathrm{O}_{3}$ : C, 65.85; $\mathrm{H}, 4.91$. Found: C, 65.98; $\mathrm{H}, 4.66$.
3-Hydroxy-5,6-dimethoxy-3-methyl-3H-isobenzofuran-1-one (4b) Yield: $1.04 \mathrm{~g}$ (98\%); white solid; $\mathrm{mp} 143-145^{\circ} \mathrm{C}$ (EtOAc).

IR ( $\mathrm{NaCl}): 3378(\mathrm{OH}), 1719(\mathrm{C}=0) \mathrm{cm}^{-1}$.

${ }^{1} \mathrm{H} \mathrm{NMR}\left(\mathrm{CDCl}_{3}\right): \delta=1.90\left(\mathrm{~s}, 3 \mathrm{H}, \mathrm{CH}_{3}\right), 3.90\left(\mathrm{~s}, 3 \mathrm{H}, \mathrm{OCH}_{3}\right), 3.98(\mathrm{~s}, 3 \mathrm{H}$, $\mathrm{OCH}_{3}$ ), 5.44 (br s, $\left.1 \mathrm{H}, \mathrm{OH}\right), 6.96(\mathrm{~s}, 1 \mathrm{H}, \mathrm{Ar}-\mathrm{H}), 7.15$ (s, $\left.1 \mathrm{H}, \mathrm{Ar}-\mathrm{H}\right)$.

${ }^{13} \mathrm{C} \mathrm{NMR}\left(\mathrm{CDCl}_{3}\right): \delta=26.3\left(\mathrm{CH}_{3}\right), 56.2\left(\mathrm{OCH}_{3}\right), 56.3\left(\mathrm{OCH}_{3}\right), 103.8(\mathrm{CH}$, C), $106.2(\mathrm{CH}), 117.9(\mathrm{C}), 143.8(\mathrm{C}), 150.8(\mathrm{C}), 154.7(\mathrm{C}), 169.0(\mathrm{C}=\mathrm{O})$. MS: $m / z(\%)=224(29)\left[\mathrm{M}^{+}\right], 209(100)$.

Anal. Calcd for $\mathrm{C}_{11} \mathrm{H}_{12} \mathrm{O}_{5}: \mathrm{C}, 58.93 ; \mathrm{H}, 5.39$. Found: C, 59.16; $\mathrm{H}, 5.26$.

3-Bromomethyl-3-hydroxy-3H-isobenzofuran-1-ones 5; General Procedure

Bromine ( $1.2 \mathrm{~mL}, 23.4 \mathrm{mmol}$ ) was added dropwise, under stirring, to solutions of lactols $\mathbf{4}$ and $\mathbf{4 b}(23.4 \mathrm{mmol})$ in a mixture of AcOH/toluene $(1: 2,90 \mathrm{~mL})$ heated at $60^{\circ} \mathrm{C}$. The reaction mixtures were stirred for 30 minutes, evaporated to dryness, and the residues were crystallised from $\mathrm{CHCl}_{3}$ to afford $\mathbf{5 a}$ and $\mathbf{5 b}$, respectively.

\section{3-Bromomethyl-3-hydroxy-3H-isobenzofuran-1-one (5a)}

Yield: $5.46 \mathrm{~g}$ (96\%); white solid; $\mathrm{mp} 115-117{ }^{\circ} \mathrm{C}\left(\mathrm{CHCl}_{3}\right)$.

IR ( $\mathrm{NaCl}): 3260(\mathrm{OH}), 1752(\mathrm{C}=0) \mathrm{cm}^{-1}$.

${ }^{1} \mathrm{H} \mathrm{NMR}\left(\mathrm{CDCl}_{3}\right): \delta=3.85\left(\mathrm{~d}, J=4.4 \mathrm{~Hz}, 2 \mathrm{H}, \mathrm{CH}_{2}\right), 5.34($ br s, $1 \mathrm{H}, \mathrm{OH})$, 7.55-7.84 (m, $4 \mathrm{H}, 4$ × Ar-H).

${ }^{13} \mathrm{C}$ NMR $\left(\mathrm{CDCl}_{3}, \mathrm{CD}_{3} \mathrm{OD}\right): \delta=35.1\left(\mathrm{CH}_{2}\right), 104.4(\mathrm{C}), 122.7(\mathrm{CH}), 125.2$ $(\mathrm{CH}), 126.9(\mathrm{C}), 130.9(\mathrm{CH}), 134.6(\mathrm{CH}), 146.9(\mathrm{C}), 168.4(\mathrm{C}=\mathrm{O})$.

MS: $m / z(\%)=245(77)[\mathrm{M}+\mathrm{H}]^{+}, 243(80)[\mathrm{M}+\mathrm{H}]^{+}, 227(98), 225$ (100).

Anal. Calcd for $\mathrm{C}_{9} \mathrm{H}_{7} \mathrm{BrO}_{3}$ : C, 44.47; $\mathrm{H}, 2.90 ; \mathrm{Br}, 32.87$. Found: $\mathrm{C}, 44.15$; $\mathrm{H}, 3.21$.

3-Bromomethyl-3-hydroxy-5,6-dimethoxy-3H-isobenzofuran-1one (5b)

Yield: $1.60 \mathrm{~g}$ (90\%); white solid; $\mathrm{mp} 132-134{ }^{\circ} \mathrm{C}\left(\mathrm{CHCl}_{3}\right)$.

IR ( $\mathrm{NaCl}): 3219(\mathrm{OH}), 1740(\mathrm{C}=\mathrm{O}) \mathrm{cm}^{-1}$.

${ }^{1} \mathrm{H} \mathrm{NMR}\left(\mathrm{CDCl}_{3}\right): \delta=3.84\left(\mathrm{~s}, 2 \mathrm{H}, \mathrm{CH}_{2}\right), 3.93\left(\mathrm{~s}, 3 \mathrm{H}, \mathrm{OCH}_{3}\right), 4.00(\mathrm{~s}, 3 \mathrm{H}$, $\left.\mathrm{OCH}_{3}\right), 7.07$ (s, $\left.1 \mathrm{H}, \mathrm{Ar}-\mathrm{H}\right), 7.20(\mathrm{~s}, 1 \mathrm{H}, \mathrm{Ar}-\mathrm{H})$.

${ }^{13} \mathrm{C} \mathrm{NMR}\left(\mathrm{CDCl}_{3}\right): \delta=36.2\left(\mathrm{CH}_{2}\right), 56.4\left(\mathrm{OCH}_{3}\right), 56.5\left(\mathrm{OCH}_{3}\right), 103.1(\mathrm{C}-\mathrm{OH})$, $104.3(\mathrm{CH}), 106.1(\mathrm{CH}), 119.0(\mathrm{C}), 140.6(\mathrm{C}), 151.7(\mathrm{C}), 155.0(\mathrm{C}), 168.2$ $(\mathrm{C}=\mathrm{O})$.

MS: $m / z(\%)=304(19)[\mathrm{M}+\mathrm{H}]^{+}, 302(20)[\mathrm{M}+\mathrm{H}]^{+}, 223(100)$

Anal. Calcd for $\mathrm{C}_{11} \mathrm{H}_{11} \mathrm{BrO}_{5}$ : C, 43.59; $\mathrm{H}, 3.66 ; \mathrm{Br}, 26.36$. Found: $\mathrm{C}$, 43.86; $\mathrm{H}, 3.81$

\section{Isochroman-1,4-diones 6; General Procedure}

$\mathrm{NaOAc}(21.40 \mathbf{m m o l})$ was added in portions to solution of $\mathbf{5 a}$ and $\mathbf{5 b}$ $(21.40 \mathrm{mmol})$ in EtOH $(11 \mathrm{~mL})$. The reaction mixtures were stirred at r.t. for $30 \mathrm{~min}$ and then were concentrated to dryness, under vacuum. The solids were washed with water and dried under vacuum to give compounds $\mathbf{6 a}$ and $\mathbf{6 b}$, respectively

\section{Isochroman-1,4-dione $(\mathbf{6 a})^{36}$}

Yield: $3.40 \mathrm{~g}$ (98\%); white solid; $\mathrm{mp} 147-148^{\circ} \mathrm{C}\left(\mathrm{CHCl}_{3}\right)$. IR (NaCl): $1725(\mathrm{C}=0), 1695(\mathrm{C}=0) \mathrm{cm}^{-1}$.

${ }^{1} \mathrm{H}$ NMR $\left(\mathrm{CDCl}_{3}\right): \delta=5.14\left(\mathrm{~s}, 2 \mathrm{H}, \mathrm{CH}_{2}\right), 7.82-7.95(\mathrm{~m}, 2 \mathrm{H}, 2 \times \mathrm{Ar}-\mathrm{H})$, 8.06-8.10 (m, $1 \mathrm{H}, \mathrm{Ar}-\mathrm{H}), 8.25-8.29$ (m, $1 \mathrm{H}, \mathrm{Ar}-\mathrm{H})$. 
${ }^{13} \mathrm{C}$ NMR $\left(\mathrm{CDCl}_{3}\right): \delta=73.3\left(\mathrm{CH}_{2}\right), 125.5(\mathrm{CH}), 127.8(\mathrm{C}), 130.7(\mathrm{CH})$, $131.7(\mathrm{C}), 134.6(\mathrm{CH}), 135.8(\mathrm{CH}), 161.4(\mathrm{C}=\mathrm{O}), 189.4(\mathrm{C}=0)$.

Anal. Calcd for $\mathrm{C}_{9} \mathrm{H}_{9} \mathrm{O}_{3}$ : C, 66.67; $\mathrm{H}, 3.73$. Found: C, 61.03; $\mathrm{H}, 3.59$.

\section{6,7-Dimethoxyisochroman-1,4-dione (6b)}

Yield: $1.18 \mathrm{~g}$ (95\%); white solid; $\mathrm{mp} 253-255{ }^{\circ} \mathrm{C}\left(\mathrm{CHCl}_{3}\right)$.

IR ( $\mathrm{NaCl}): 1714(\mathrm{C}=0), 1683(\mathrm{C}=0) \mathrm{cm}^{-1}$.

${ }^{1} \mathrm{H} \mathrm{NMR}\left(\mathrm{CDCl}_{3}\right): \delta=4.04\left(\mathrm{~s}, 3 \mathrm{H}, \mathrm{OCH}_{3}\right), 4.06\left(\mathrm{~s}, 3 \mathrm{H}, \mathrm{OCH}_{3}\right), 5.11(\mathrm{~s}$, $\left.2 \mathrm{H}, \mathrm{CH}_{2}\right), 7.47$ (s, $\left.1 \mathrm{H}, \mathrm{Ar}-\mathrm{H}\right), 7.66(\mathrm{~s}, 1 \mathrm{H}, \mathrm{Ar}-\mathrm{H})$.

${ }^{13} \mathrm{C} \mathrm{NMR}\left(\mathrm{CDCl}_{3}\right): \delta=56.6\left(\mathrm{OCH}_{3}\right), 56.8\left(\mathrm{OCH}_{3}\right), 73.4\left(\mathrm{CH}_{2}\right), 106.1(\mathrm{CH})$, $111.4(\mathrm{CH}), 122.6(\mathrm{C}), 126.5(\mathrm{C}), 154.2(\mathrm{C}), 155.2(\mathrm{C}), 161.6(\mathrm{C}=0)$, $188.6(\mathrm{C}=\mathrm{O})$.

MS: $m / z(\%)=222(34)\left[\mathrm{M}^{+}\right], 164(100)$.

Anal. Calcd for $\mathrm{C}_{11} \mathrm{H}_{10} \mathrm{O}_{5}$ : C, 59.46; $\mathrm{H}, 4.54$. Found: $\mathrm{C}, 59.67 ; \mathrm{H}, 4.23$.

\section{3-Benzylidene-isochromane-1,4-diones 8; General Procedure}

$\mathrm{NH}_{4} \mathrm{OAc}$ (209 mg, $2.71 \mathrm{mmol}$ ) was added to a solution of $\mathbf{6 a}$ and $\mathbf{6 b}$ $(1.23 \mathrm{mmol})$ and the respective aldehyde $(1.23 \mathrm{mmol})$ in $\mathrm{AcOH}(5$ $\mathrm{mL}$ ), and the resulting solutions were heated at $60^{\circ} \mathrm{C}$ for $6 \mathrm{~h}$. The reaction mixtures were allowed to cool to r.t., water $(10 \mathrm{~mL})$ was then added and the precipitated solids were washed with water and dried, to give $\mathbf{8 a}, \mathbf{8 b}$ or $\mathbf{1 2}$

\section{3-(2-Bromobenzylidene)isochroman-1,4-dione (8a)}

Yield: $988 \mathrm{mg}$ (81\%); yellow solid; $\mathrm{mp} 130-132{ }^{\circ} \mathrm{C}(\mathrm{MeOH})$.

IR ( $\mathrm{NaCl}): 1746(\mathrm{C}=0) \mathrm{cm}^{-1}$.

${ }^{1} \mathrm{H} \mathrm{NMR}\left(\mathrm{CDCl}_{3}\right): \delta=7.19-7.29(\mathrm{~m}, 1 \mathrm{H}, \mathrm{Ar}-\mathrm{H}), 7.38-7.46(\mathrm{~m}, 1 \mathrm{H}, \mathrm{Ar}-$ H), 7.58-7.69 (m, $2 \mathrm{H},=\mathrm{CH}, \mathrm{Ar}-\mathrm{H}), 7.86-7.93(\mathrm{~m}, 2 \mathrm{H}, 2 \times \mathrm{Ar}-\mathrm{H}), 8.24-$ $8.36(\mathrm{~m}, 3 \mathrm{H}, 3 \times \mathrm{Ar}-\mathrm{H})$.

${ }^{13} \mathrm{C}$ NMR $\left(\mathrm{CDCl}_{3}\right): \delta=118.1(\mathrm{CH}), 126.6(\mathrm{C}), 126.7(\mathrm{C}), 126.9(\mathrm{CH})$, $127.7(\mathrm{CH}), 130.6(\mathrm{CH}), 131.2(\mathrm{CH}), 131.5(\mathrm{C}), 132.5(\mathrm{CH}), 133.1(\mathrm{C})$, 133.2 $(\mathrm{CH}), 135.2(2 \times \mathrm{CH}), 145.4(\mathrm{C}), 157.8(\mathrm{C}=\mathrm{O}), 176.6(\mathrm{C}=0)$.

MS: $m / z(\%)=328(44)\left[\mathrm{M}^{+}\right], 330(39)\left[\mathrm{M}^{+}\right], 249(100)$.

HRMS (ESI): $m / z[\mathrm{M}+\mathrm{H}]^{+}$calcd for $\mathrm{C}_{16} \mathrm{H}_{10} \mathrm{BrO}_{3}$ : 328.9808; found: 328.9813.

\section{3-(2-Bromo-4,5-dimethoxybenzylidene)-6,7-dimethoxyiso-chro- man-1,4-dione (8b)}

Yield: $342 \mathrm{mg}$ (85\%); yellow solid; $\mathrm{mp} 289-290{ }^{\circ} \mathrm{C}(\mathrm{MeOH})$.

IR $(\mathrm{NaCl}): 1734(\mathrm{C}=\mathrm{O}) \mathrm{cm}^{-1}$.

${ }^{1} \mathrm{H} \mathrm{NMR}\left(\mathrm{CDCl}_{3}\right): \delta=3.94\left(\mathrm{~s}, 3 \mathrm{H}, \mathrm{OCH}_{3}\right), 4.00\left(\mathrm{~s}, 3 \mathrm{H}, \mathrm{OCH}_{3}\right), 4.07(\mathrm{~s}$, $\left.6 \mathrm{H}, 2 \times \mathrm{OCH}_{3}\right), 7.12,7.27,7.58,7.65,8.08(5 \mathrm{~s}, 5 \mathrm{H}, 4 \times \mathrm{Ar}-\mathrm{H},=\mathrm{CH})$.

MS: $m / z(\%)=450(10)\left[\mathrm{M}^{+}\right], 448(10)\left[\mathrm{M}^{+}\right], 369(100)$.

HRMS (ESI): $m / z[\mathrm{M}+\mathrm{H}]^{+}$calcd for $\mathrm{C}_{20} \mathrm{H}_{18} \mathrm{BrO}_{7}$ : 449.0230; found: 449.0219.

\section{3-(2-Carbomethoxybenzylidene)isochromane-1,4-dione (11)}

Yield: $284 \mathrm{~g}$ (75\%); yellow solid; mp $227-229{ }^{\circ} \mathrm{C}(\mathrm{MeOH})$.

IR (NaCl): $1763(\mathrm{C}=0) \mathrm{cm}^{-1}$.

${ }^{1} \mathrm{H}$ NMR $\left(\mathrm{CDCl}_{3}\right): \delta=3.90\left(\mathrm{~s}, 3 \mathrm{H}, \mathrm{CH}_{3}\right), 7.42(\mathrm{t}, J=7.5 \mathrm{~Hz}, 1 \mathrm{H}, \mathrm{Ar}-\mathrm{H})$, $7.59(\mathrm{t}, J=7.5 \mathrm{~Hz}, 1 \mathrm{H}, \mathrm{H}-\mathrm{Ar}), 7.81-7.91(\mathrm{~m}, 3 \mathrm{H}, \mathrm{H}-\mathrm{C}=$ and $2 \times \mathrm{H}-\mathrm{Ar})$, $7.96(\mathrm{~d}, J=7.7 \mathrm{~Hz}, 1 \mathrm{H}, \mathrm{Ar}-\mathrm{H}), 8.07$ (d, $J=7.7 \mathrm{~Hz}, 1 \mathrm{H}, \mathrm{Ar}-\mathrm{H}), 8.19-8.31$ ( $\mathrm{m}, 2 \mathrm{H}, 2 \times \mathrm{Ar}-\mathrm{H})$.
${ }^{13} \mathrm{C} \mathrm{NMR}\left(\mathrm{CDCl}_{3}\right): \delta=52.3\left(\mathrm{CH}_{3}\right), 118.9(\mathrm{CH}=\mathrm{C}), 126.7(\mathrm{CH}-\mathrm{Ar}), 126.8$ (C-Ar), 129.2 (CH-Ar), 130.4 (CH-Ar), 130.6 (CH-Ar), 131.7 (CH-Ar), 131.8 (C-Ar), 132.1 (CH-Ar), 132.2 (C-Ar), 133.1 (C-Ar), $135.0(2 \times \mathrm{CH})$, 144.7 (C=CH), $157.9(\mathrm{CO}), 167.1(\mathrm{CO}), 176.7(\mathrm{CO})$.

MS: $m / z(\%)=309.3(53)[\mathrm{M}+\mathrm{H}]^{+}, 174.2(100)\left[\mathrm{M}-\mathrm{C}_{8} \mathrm{H}_{7} \mathrm{O}_{2}\right]$.

HRMS (ESI): $m / z[M+H]^{+}$calcd for $\mathrm{C}_{18} \mathrm{H}_{13} \mathrm{O}_{5}: 309.3702$; found 309.3706.

\section{2-Phenyl-3-hydroxynaphthalene-1,4-diones 10; General Proce- dure}

A solution of $\mathrm{NaOMe}(56.7 \mathrm{mg}, 1.05 \mathrm{mmol})$ in $\mathrm{MeOH}(15 \mathrm{~mL})$ was added dropwise to stirred solutions of benzylideneisochromane-1,4-dione $8 \mathbf{a}$ and $8 \mathbf{b}(0.5 \mathrm{mmol})$ in $\mathrm{MeOH}(20 \mathrm{~mL})$ cooled at $0{ }^{\circ} \mathrm{C}$. The resulting solutions were stirred for $24 \mathrm{~h}$ at r.t., poured into water $(15 \mathrm{~mL})$ and acidified with aqueous $1 \mathrm{M} \mathrm{HCl}$. The mixtures were extracted with $\mathrm{CH}_{2} \mathrm{Cl}_{2}(3 \times 10 \mathrm{~mL})$ and the combined organic layers were dried with anhydrous $\mathrm{Na}_{2} \mathrm{SO}_{4}$ and concentrated to dryness under vacuum. The residues were purified by recrystallisation from $\mathrm{MeOH}$, to yield 10a, 10b or 15.

\section{2-(2-Bromophenyl)-3-hydroxy-1,4-naphthoquinone (10a)}

Yield: $325 \mathrm{mg}$ (65\%); red solid; mp $172-174{ }^{\circ} \mathrm{C}(\mathrm{MeOH})$.

IR (NaCl): $3425(\mathrm{OH}), 1675(\mathrm{C}=0), 1641(\mathrm{C}=0) \mathrm{cm}^{-1}$.

${ }^{1} \mathrm{H} \mathrm{NMR}\left(\mathrm{CDCl}_{3}\right): \delta=7.24-7.31(\mathrm{~m}, 2 \mathrm{H}, 2 \times \mathrm{Ar}-\mathrm{H}), 7.37-7.45(\mathrm{~m}, 1 \mathrm{H}$, Ar-H), 7.66-7.85 (m, 3 H, $3 \times$ Ar-H), 8.15-8.21 (m, 2 H, $2 \times$ Ar-H).

${ }^{13} \mathrm{C} \mathrm{NMR}\left(\mathrm{CDCl}_{3}\right): \delta=122.6(\mathrm{C}), 123.9(\mathrm{C}), 126.3(\mathrm{CH}), 127.1(\mathrm{CH})$, $127.3(\mathrm{CH}), 129.3(\mathrm{C}), 130.1(\mathrm{CH}), 131.4(\mathrm{CH}), 131.8(\mathrm{C}), 132.7(\mathrm{C}, \mathrm{CH})$, 133.2 (CH), $135.4(\mathrm{CH}), 152.7(\mathrm{C}), 181.6(\mathrm{C}=\mathrm{O}), 182.7(\mathrm{C}=\mathrm{O})$.

MS: $m / z(\%)=331(95)[\mathrm{M}+\mathrm{H}]^{+}, 329(100)[\mathrm{M}+\mathrm{H}]^{+}$.

HRMS (ESI): $m / z$ [M $+\mathrm{H}]^{+}$calcd for $\mathrm{C}_{16} \mathrm{H}_{10} \mathrm{BrO}_{3}$ : 328.9808; found: 328.9795 .

2-(2-Bromo-4,5-dimethoxyphenyl)-3-hydroxy-6,7-dimethoxy-1,4naphthoquinone (10b)

Yield: $170 \mathrm{mg}$ (85\%); red solid; $\mathrm{mp} 222-224^{\circ} \mathrm{C}(\mathrm{MeOH})$.

3-(2-Carbomethoxybenzoyl)-1H-isochromen-1-one (14)

Yield: $690 \mathrm{mg}$ (70\%); red solid; $\mathrm{mp} 139-141{ }^{\circ} \mathrm{C}\left(\mathrm{MeOH} / \mathrm{CH}_{2} \mathrm{Cl}_{2}\right)$.

IR ( $\mathrm{NaCl}): 1765$ (CO) $\mathrm{cm}^{-1}$.

${ }^{1} \mathrm{H} \mathrm{NMR}\left(\mathrm{CDCl}_{3}\right): \delta=3.79\left(\mathrm{~s}, 3 \mathrm{H}, \mathrm{CH}_{3}\right), 7.33(\mathrm{~s}, 1 \mathrm{H}, \mathrm{Ar}-\mathrm{H}), 7.41-7.49$ $(\mathrm{m}, 1 \mathrm{H}, \mathrm{H}-\mathrm{Ar}) ; 7.55-7.72(\mathrm{~m}, 4 \mathrm{H}, \mathrm{H}-\mathrm{C}=$ and $3 \times \mathrm{Ar}-\mathrm{H}), 7.77(\mathrm{t}$, $J=7.5 \mathrm{~Hz}, 1 \mathrm{H}, \mathrm{Ar}-\mathrm{H}), 8.07(\mathrm{~d}, J=7.7 \mathrm{~Hz}, 1 \mathrm{H}, \mathrm{Ar}-\mathrm{H}), 8.31(\mathrm{~d}, J=7.7 \mathrm{~Hz}$, $1 \mathrm{H}, \mathrm{Ar}-\mathrm{H})$

${ }^{13} \mathrm{C} \mathrm{NMR}\left(\mathrm{CDCl}_{3}\right): \delta=52.6\left(\mathrm{CH}_{3}\right), 110.5(\mathrm{CH}=\mathrm{C}), 122.7(\mathrm{C}-\mathrm{Ar}), 127.9$ (CH-Ar), 128.1 (CH-Ar), 129.4 (C-Ar), 130.0 (C-Ar), 130.5 (CH-Ar), 131.8 (CH-Ar), 130.7 (CH-Ar), 132.2 (CH-Ar), 132.8 (CH-Ar), 135.1 (C$\mathrm{Ar}), 139.4(\mathrm{CH}-\mathrm{Ar}), 149.6$ (C = CH), $160.4(\mathrm{CO}), 166.3(\mathrm{CO}), 189.3(\mathrm{CO})$. MS: $m / z(\%)=309(65)[\mathrm{M}+\mathrm{H}]^{+}, 174(100)\left[\mathrm{M}-\mathrm{C}_{8} \mathrm{H}_{7} \mathrm{O}_{2}\right]$.

HRMS (ESI): $m / z[M+H]^{+}$calcd for $\mathrm{C}_{18} \mathrm{H}_{13} \mathrm{O}_{5}: 309.3523$; found: 309.3525 .

\section{o-Acetylphenylacetic Acids 17; General Procedure}

$20 \%$ Aqueous sulphuric acid (3.5 mL) was added, under nitrogen, to a solution of compounds $\mathbf{3 c}$ and $\mathbf{3 d}(1.56 \mathrm{mmol})$ in dioxane $(9.0 \mathrm{~mL})$ and the mixtures were heated at reflux for $4 \mathrm{~h}$. The reaction mixtures were added over $10 \%$ aqueous $\mathrm{NaOH}(2 \times 15 \mathrm{~mL})$ and extracted with $\mathrm{Et}_{2} \mathrm{O}(10 \mathrm{~mL})$. The aqueous extracts were cooled at $0{ }^{\circ} \mathrm{C}$ and $20 \% \mathrm{H}_{2} \mathrm{SO}_{4}$ 
was added until pH 3.0. The suspensions were extracted with $\mathrm{CHCl}_{3}$ $(3 \times 20 \mathrm{~mL})$. The combined extracts were washed with water $(15 \mathrm{~mL})$, dried with anhydrous $\mathrm{Na}_{2} \mathrm{SO}_{4}$ and concentrated to dryness under vacuum, to give residues that were purified by recrystallisation, to afford compounds $\mathbf{1 7} \mathbf{a}$ or $\mathbf{1 7 b}$

\section{2-(2-Acetylphenyl)acetic Acid (16a)}

Yield: $255 \mathrm{mg}$ (92\%); mp $135{ }^{\circ} \mathrm{C}(\mathrm{MeOH})$.

IR (NaCl): $3440(\mathrm{COOH}), 1707(\mathrm{C}=0), 1671(\mathrm{C}=0) \mathrm{cm}^{-1}$.

${ }^{1} \mathrm{H}$ NMR $\left(\mathrm{CDCl}_{3}, \mathrm{CD}_{3} \mathrm{OD}\right): \delta=2.50\left(\mathrm{~s}, 3 \mathrm{H}, \mathrm{CH}_{3}\right), 3.81\left(\mathrm{~s}, 2 \mathrm{H}, \mathrm{CH}_{2}\right), 4.23$ (s, $1 \mathrm{H}, \mathrm{COOH}), 7.17$ (d, J=7.3 Hz, $1 \mathrm{H}, \mathrm{Ar}-\mathrm{H}), 7.29-7.41(\mathrm{~m}, 2 \mathrm{H}$, $2 \times \mathrm{Ar}-\mathrm{H}), 7.73(\mathrm{~d}, J=7.5 \mathrm{~Hz}, 1 \mathrm{H}, \mathrm{Ar}-\mathrm{H})$.

${ }^{13} \mathrm{C} \operatorname{NMR}\left(\mathrm{CDCl}_{3}\right): \delta=28.3\left(\mathrm{CH}_{3}\right), 39.9\left(\mathrm{CH}_{3}\right), 127.8(\mathrm{CH}), 132.0(\mathrm{CH})$, $132.5(\mathrm{CH}), 134.3(\mathrm{C}), 136.93(\mathrm{C}), 173.8(\mathrm{C}=\mathrm{O}), 202.4(\mathrm{C}=\mathrm{O})$.

MS: $m / z(\%)=178(13)\left[\mathrm{M}^{+}\right], 160(28), 135(85), 132(100)$.

Anal. Calcd for $\mathrm{C}_{10} \mathrm{H}_{10} \mathrm{O}_{3}$ : C, 67.41; H, 5.66. Found: C, 67.26; H, 5.78.

\section{2-(2-Acetyl-4,5-dimethoxyphenyl)acetic Acid (16b)}

Yield: $461 \mathrm{mg}$ (86\%); mp $172-173{ }^{\circ} \mathrm{C}(\mathrm{MeOH})$.

IR ( $\mathrm{NaCl}): 3020(\mathrm{COOH}), 1730(\mathrm{C}=0), 1638(\mathrm{C}=0) \mathrm{cm}^{-1}$.

${ }^{1} \mathrm{H}$ NMR $\left(\mathrm{CDCl}_{3}, \mathrm{CD}_{3} \mathrm{OD}\right): \delta=2.60\left(\mathrm{~s}, 3 \mathrm{H}, \mathrm{CH}_{3}\right), 3.87\left(\mathrm{~s}, 3 \mathrm{H}, \mathrm{CH}_{3}\right), 3.97$ (s, $\left.6 \mathrm{H}, 2 \times \mathrm{OCH}_{3}\right), 6.78(\mathrm{~s}, 1 \mathrm{H}, \mathrm{Ar}-\mathrm{H}), 7.35(\mathrm{~s}, 1 \mathrm{H}, \mathrm{Ar}-\mathrm{H})$.

${ }^{13} \mathrm{C} \mathrm{NMR}\left(\mathrm{CDCl}_{3}, \mathrm{CD}_{3} \mathrm{OD}\right): \delta=28.2\left(\mathrm{CH}_{3}\right), 40.2\left(\mathrm{CH}_{3}\right), 55.7\left(\mathrm{OCH}_{3}\right), 55.9$ $\left(\mathrm{OCH}_{3}\right), 113.5(\mathrm{CH}), 115.1(\mathrm{CH}), 128.6(\mathrm{C}), 129.5(\mathrm{C}), 147.0\left(\mathrm{Ar}-\mathrm{OCH}_{3}\right)$, $151.8\left(\mathrm{Ar}-\mathrm{OCH}_{3}\right), 173.7(\mathrm{C}=\mathrm{O}), 200.3(\mathrm{C}=\mathrm{O})$.

MS: $m / z(\%)=238(13)\left[\mathrm{M}^{+}\right], 185(39), 179(100)$.

Anal. Calcd for $\mathrm{C}_{12} \mathrm{H}_{14} \mathrm{O}_{5}$ : C, 60.50; H, 5.92. Found: C, 60.23; H, 6.12.

\section{Methyl 2-(2-Cinnamoylphenyl)acetates 17; General Procedure}

$15 \%$ Aqueous $\mathrm{KOH}(2 \mathrm{~mL}$ ) was added to stirred solutions of $\mathbf{1 6 a}$ and 16b $(0.90 \mathrm{mmol})$ and the appropriate aldehyde $(1.10 \mathrm{mmol})$ in $\mathrm{EtOH}$ $(5 \mathrm{~mL})$. The mixtures were heated at reflux for $45 \mathrm{~min}$, cooled and acidified with $10 \%$ aqueous $\mathrm{HCl}(10 \mathrm{~mL})$. The suspensions were extracted with $\mathrm{CHCl}_{3}(3 \times 15 \mathrm{~mL})$. The combined extracts were washed with water $(3 \times 15 \mathrm{~mL})$, dried with anhydrous $\mathrm{Na}_{2} \mathrm{SO}_{4}$ and concentrated to dryness under vacuum, to give a yellow oil, that were dissolved in $\mathrm{MeOH}(20 \mathrm{~mL})$. A catalytic amount of $p$-toluensulphonic acid was added and the mixtures were stirred at r.t. for 1.5 days. The reaction mixtures were concentrated under vacuum and the remaining oils were purified by flash column chromatography, using $\mathrm{CH}_{2} \mathrm{Cl}_{2}$ as eluent, to provide 17a-d.

\section{Methyl 2-(2-Cinnamoylphenyl)acetate (17a)}

Yield: $127 \mathrm{mg}$ (50\%); colourless oil.

IR (NaCl): $1735(\mathrm{C}=0), 1723(\mathrm{C}=0), 1662(\mathrm{C}=0), 1636(\mathrm{C}=0) \mathrm{cm}^{-1}$.

${ }^{1} \mathrm{H} \mathrm{NMR}\left(\mathrm{CDCl}_{3}\right): \delta=3.63\left(\mathrm{~s}, 2 \mathrm{H}, \mathrm{CH}_{2}\right), 3.90\left(\mathrm{~s}, 3 \mathrm{H}, \mathrm{OCH}_{3}\right), 7.20-7.50$ (m, $7 \mathrm{H}, \mathrm{Ar}-\mathrm{H}), 7.53-7.70(\mathrm{~m}, 1 \mathrm{H}, \mathrm{Ar}-\mathrm{H})$.

${ }^{13} \mathrm{C} \mathrm{NMR}\left(\mathrm{CDCl}_{3}\right): \delta=38.9\left(\mathrm{CH}_{3}\right), 51.8\left(\mathrm{OCH}_{3}\right), 125.3(\mathrm{CH}), 126.96(\mathrm{CH})$, $128.3(2 \times \mathrm{CH}), 128.8(2 \times \mathrm{CH}), 128.9(\mathrm{CH}), 130.5(\mathrm{Ar}-\mathrm{H}), 131.0(\mathrm{CH})$, $132(\mathrm{CH}), 133.7(\mathrm{C}), 134.4(\mathrm{C}), 138.44(\mathrm{C}), 145.7(\mathrm{CH}), 171.7(\mathrm{C}=\mathrm{O})$, $194.7(\mathrm{C}=0)$.

MS: $m / z(\%)=280(6)\left[\mathrm{M}^{+}\right], 220(100)$.

Anal. Calcd for $\mathrm{C}_{18} \mathrm{H}_{16} \mathrm{O}_{3}$ : C, 77.12; $\mathrm{H}, 5.75$. Found: C, 77.10; $\mathrm{H}, 5.83$.
Methyl (E)-2-(2-(3-(3,4-dimethoxyphenyl)acryloyl)phenyl)acetate (17b)

Yield: $363 \mathrm{mg}$ (95\%); colourless oil.

IR ( $\mathrm{NaCl}): 1734(\mathrm{C}=0) ; 1656(\mathrm{C}=0), 1632(\mathrm{C}=0) \mathrm{cm}^{-1}$.

${ }^{1} \mathrm{H}$ NMR $\left(\mathrm{CDCl}_{3}, \mathrm{CD}_{3} \mathrm{OD}\right): \delta=3.63\left(\mathrm{~s}, 2.7 \mathrm{H}, \mathrm{OCH}_{3}\right), 3.65(\mathrm{~s}, 2.7 \mathrm{H}$, $\left.\mathrm{OCH}_{3}\right)$, 3.87-3.92(m, 9.5 H, $\left.2 \times \mathrm{CH}_{2}+4 \times \mathrm{OCH}_{3}\right), 6.70-6.90(\mathrm{~m}, 2.3 \mathrm{H}$, Ar-H), 7.00-7.19 (m, 3.5 H, Ar-H), 7.19-7.51 (m, 4.8 H, Ar-H), 7.53$7.69(\mathrm{~m}, 1.9 \mathrm{H}, \mathrm{Ar}-\mathrm{H})$.

${ }^{13} \mathrm{C} \mathrm{NMR}\left(\mathrm{CDCl}_{3}, \mathrm{CD}_{3} \mathrm{OD}\right): \delta=38.8\left(\mathrm{CH}_{3}\right), 51.8\left(\mathrm{OCH}_{3}\right), 55.69\left(\mathrm{OCH}_{3}\right)$, $55.7\left(\mathrm{OCH}_{3}\right), 55.8\left(\mathrm{OCH}_{3}\right), 55.8\left(\mathrm{OCH}_{3}\right), 108.9(\mathrm{CH}), 109.7(\mathrm{Ar}-\mathrm{H}), 110.9$ $(\mathrm{Ar}-\mathrm{H}), 111.0(\mathrm{CH}), 121.4(\mathrm{CH}), 123.2(\mathrm{CH}), 123.5(\mathrm{CH}), 124.7(\mathrm{CH})$, $126.9(\mathrm{CH}), 127.4(\mathrm{C}), 127.8(\mathrm{CH}), 128.6(\mathrm{CH}), 128.9(\mathrm{CH}), 130.8(\mathrm{CH})$, $131.9(\mathrm{CH}), 133.4(\mathrm{C}), 133.5(\mathrm{C}), 138.7(\mathrm{C}), 138.8(\mathrm{C}), 141.9(\mathrm{CH}), 146.0$ (CH), $146.3(\mathrm{CH}), 149.0$ (C), 149.1 (C), 150.2 (C), 151.3 (C), 171.7 $(\mathrm{C}=0), 194.8(\mathrm{C}=0), 194.9(\mathrm{C}=0)$.

MS: $m / z(\%)=340(22)\left[\mathrm{M}^{+}\right], 281(26), 164(24), 151$ (100).

Anal. Calcd for $\mathrm{C}_{20} \mathrm{H}_{20} \mathrm{O}_{5}: \mathrm{C}, 70.58 ; \mathrm{H}, 5.92$. Found: C, 70.23; H, 6.17.

\section{Methyl 2-(2-Cinnamoyl-4,5-dimethoxyphenyl)acetate (17c)}

Yield: $184 \mathrm{mg}$ (87\%); colourless oil.

IR ( $\mathrm{NaCl}): 1734(\mathrm{C}=0), 1655(\mathrm{C}=0)$.

${ }^{1} \mathrm{H}$ NMR $\left(\mathrm{CDCl}_{3}, \mathrm{CD}_{3} \mathrm{OD}\right): \delta=3.68\left(\mathrm{~s}, 3 \mathrm{H}, \mathrm{CH}_{3}\right), 3.86\left(\mathrm{~s}, 2 \mathrm{H}, \mathrm{CH}_{2}\right), 3.92$ (s, $\left.3 \mathrm{H}, \mathrm{OCH}_{3}\right) ; 3.95\left(\mathrm{~s}, 3 \mathrm{H}, \mathrm{OCH}_{3}\right), 6.82(\mathrm{~s}, 1 \mathrm{H}, \mathrm{Ar}-\mathrm{H}), 7.21-7.31(\mathrm{~m}$, 2 H, Ar-H), 7.39-7.43 (m, 3 H, Ar-H), 7.57-7.67 (m, 3 H, Ar-H).

${ }^{13} \mathrm{C}$ NMR $\left(\mathrm{CDCl}_{3}, \mathrm{CD}_{3} \mathrm{OD}\right): \delta=39.1\left(\mathrm{CH}_{3}\right), 51.9\left(\mathrm{OCH}_{3}\right), 56.0\left(\mathrm{OCH}_{3}\right)$, $56.2\left(\mathrm{OCH}_{3}\right), 112.5(\mathrm{CH}), 114.8(\mathrm{CH}), 125.2(\mathrm{CH}), 128.4(2 \times \mathrm{CH}), 128.9$ (2× Ar-H), 130.5 (Ar-H), 130.8 (C), 134.7 (C), 145.1 (Ar-H), 147.4 (Ar$\left.\mathrm{OCH}_{3}\right), 151.2\left(\mathrm{Ar}-\mathrm{OCH}_{3}\right), 173.1(\mathrm{C}=\mathrm{O}), 193.1(\mathrm{C}=\mathrm{O})$.

MS: $m / z(\%)=340(13)\left[\mathrm{M}^{+}\right], 281(100)$.

Anal. Calcd for $\mathrm{C}_{20} \mathrm{H}_{20} \mathrm{O}_{5}: \mathrm{C}, 70.58 ; \mathrm{H}, 5.92$. Found: C, 70.72; H, 5.61.

Methyl (E)-2-(2-(3-(3,4-Dimethoxyphenyl)acryloyl)-4,5-dimethoxyphenyl)acetate (17d)

This product was not isolated; it was directly used in the next step.

Methyl 2-(2-(3-Phenylpropanoyl)phenyl)acetates 18; General Procedure

A Raney-nickel $\mathrm{H}_{2} \mathrm{O}$ suspension (ca. $50 \%$ w/w) was added over degassed solutions of 17a, 17b, 17c and 17d $(0.45 \mathrm{mmol})$ in EtOAc $(20$ $\mathrm{mL}$ ) and the resulting suspensions were purged with hydrogen and stirred at $0{ }^{\circ} \mathrm{C}$ under hydrogen ( $\left.1 \mathrm{~atm}\right)$ for $30 \mathrm{~min}$, then at r.t. for a further 30 min. Hydrogen was then removed by purging with nitrogen and the metallic catalyst was filtered off over a Celite pad that was washed with EtOAc. The solvents were removed under vacuum, to afford the respective products $\mathbf{1 8 a - d}$, as chromatographically pure oils.

\section{Methyl 2-(2-(3-Phenylpropanoyl)phenyl)acetate (18a)}

Yield: $121 \mathrm{mg}$ (93\%); colourless oil.

IR ( $\mathrm{NaCl}): 1735(\mathrm{C}=0), 1682(\mathrm{C}=0) \mathrm{cm}^{-1}$.

${ }^{1} \mathrm{H} \mathrm{NMR}\left(\mathrm{CDCl}_{3}\right): \delta=2.97-3.06\left(\mathrm{~m}, 2 \mathrm{H}, \mathrm{CH}_{2}\right), 3.23-3.31\left(\mathrm{~m}, 2 \mathrm{H}, \mathrm{CH}_{2}\right)$, $3.68\left(\mathrm{~s}, 3 \mathrm{H}, \mathrm{OCH}_{3}\right), 3.92\left(\mathrm{~s}, 2 \mathrm{H}, \mathrm{CH}_{2}\right), 7.15-7.38(\mathrm{~m}, 7 \mathrm{H}, \mathrm{Ar}-\mathrm{H}), 7.44$ (dt, $J=1.15,7.4 \mathrm{~Hz}, 1 \mathrm{H}, \mathrm{Ar}-\mathrm{H}), 7.74$ (dd, $J=1.4,7.6 \mathrm{~Hz}, 1 \mathrm{H}, \mathrm{Ar}-\mathrm{H})$.

${ }^{13} \mathrm{C} \mathrm{NMR}\left(\mathrm{CDCl}_{3}\right): \delta=30.1\left(\mathrm{CH}_{2}\right), 39.9\left(\mathrm{CH}_{2}\right), 42.4\left(\mathrm{CH}_{2}\right), 51.8\left(\mathrm{OCH}_{3}\right)$, $126.0(\mathrm{CH}), 127.3(\mathrm{CH}), 128.3(2 \times \mathrm{CH}), 128.4(2 \times \mathrm{CH}), 129.0(\mathrm{CH})$, $131.7(\mathrm{CH}), 132.6(\mathrm{CH}), 134.1(\mathrm{C}), 137.2(\mathrm{C}), 141.1(\mathrm{C}), 171.9(\mathrm{C}=\mathrm{O})$, $202.5(\mathrm{C}=0)$

MS: $m / z(\%)=282(100)\left[\mathrm{M}^{+}\right]$. 
Anal. Calcd for $\mathrm{C}_{18} \mathrm{H}_{18} \mathrm{O}_{3}$ : C, 76.57; $\mathrm{H}, 6.43$. Found: C, 76.71; $\mathrm{H}, 6.64$.

\section{Methyl 2-(2-(3-(3,4-Dimethoxyphenyl)propanoyl)phenyl)acetate (18b)}

Yield: $328, \mathrm{mg}(87 \%)$; colourless oil.

IR (NaCl): $1734(\mathrm{C}=0), 1684(\mathrm{C}=0) \mathrm{cm}^{-1}$.

${ }^{1} \mathrm{H} \mathrm{NMR}\left(\mathrm{CDCl}_{3}\right): \delta=2.97\left(\mathrm{t}, J=7.5 \mathrm{~Hz}, 2 \mathrm{H}, \mathrm{CH}_{2}\right), 3.25(\mathrm{t}, J=7.5 \mathrm{~Hz}$, $\left.2 \mathrm{H}, \mathrm{CH}_{2}\right), 3.48\left(\mathrm{~s}, 3 \mathrm{H}, \mathrm{OCH}_{3}\right), 3.86\left(\mathrm{~s}, 3 \mathrm{H}, \mathrm{OCH}_{3}\right), 3.87\left(\mathrm{~s}, 3 \mathrm{H}, \mathrm{CH}_{3}\right)$, $3.91\left(\mathrm{~s}, 2 \mathrm{H}, \mathrm{CH}_{2}\right), 6.78$ (s, $\left.3 \mathrm{H}, \mathrm{Ar}-\mathrm{H}\right), 7.25$ (d, J= 7.2 Hz, $\left.1 \mathrm{H}, \mathrm{Ar}-\mathrm{H}\right)$, 7.30-7.49 (m, $2 \mathrm{H}, \mathrm{Ar}-\mathrm{H}), 7.75$ (d, J= $7.5 \mathrm{~Hz}, 1 \mathrm{H}, \mathrm{Ar}-\mathrm{H})$.

${ }^{13} \mathrm{C}$ NMR $\left(\mathrm{CDCl}_{3}\right): \delta=29.6\left(\mathrm{CH}_{2}\right), 39.7\left(\mathrm{CH}_{2}\right), 42.5\left(\mathrm{CH}_{2}\right), 51.7\left(\mathrm{OCH}_{3}\right)$, $55.6\left(\mathrm{OCH}_{3}\right), 55.7\left(\mathrm{OCH}_{3}\right), 111.7(\mathrm{CH}), 111.6(\mathrm{CH}), 120.0(\mathrm{CH}), 127.2$ (CH), 128.9 (Ar-H), 131.6 (CH), $132.5(\mathrm{CH}), 133.7(\mathrm{C}), 134.0(\mathrm{C}), 147.2$ $\left(\mathrm{Ar}-\mathrm{OCH}_{3}\right), 148.7\left(\mathrm{Ar}-\mathrm{OCH}_{3}\right), 171.8(\mathrm{C}=\mathrm{O}), 202.5(\mathrm{C}=\mathrm{O})$

MS: $m / z(\%)=342(26)\left[\mathrm{M}^{+}\right], 151(100)$.

Anal. Calcd for $\mathrm{C}_{20} \mathrm{H}_{22} \mathrm{O}_{5}$ : C, 70.16; $\mathrm{H}, 6.48$. Found: C, 70.35; $\mathrm{H}, 6.63$.

\section{Methyl 2-(4,5-Dimethoxy-2-(3-phenylpropanoyl)phenyl)acetate (18c)}

Yield: $225 \mathrm{mg}$ (94\%); colourless oil.

IR (NaCl): $1733(\mathrm{C}=0), 1672(\mathrm{C}=0) \mathrm{cm}^{-1}$.

${ }^{1} \mathrm{H} \mathrm{NMR}\left(\mathrm{CDCl}_{3}\right): \delta=2.98-3.07\left(\mathrm{~m}, 2 \mathrm{H}, \mathrm{CH}_{2}\right), 3.19-3.27\left(\mathrm{~m}, 2 \mathrm{H}, \mathrm{CH}_{2}\right)$, $3.70\left(\mathrm{~s}, 3 \mathrm{H}, \mathrm{OCH}_{3}\right), 3.86\left(\mathrm{~s}, 3 \mathrm{H}, \mathrm{OCH}_{3}\right), 3.89\left(\mathrm{~s}, 2 \mathrm{H}, \mathrm{CH}_{2}\right), 3.92(\mathrm{~s}, 3 \mathrm{H}$, $\left.\mathrm{OCH}_{3}\right), 6.72(\mathrm{~s}, 1 \mathrm{H}, \mathrm{Ar}-\mathrm{H}), 7.16-7.38$ (m, $\left.6 \mathrm{H}, \mathrm{Ar}-\mathrm{H}\right)$.

${ }^{13} \mathrm{C} \mathrm{NMR}\left(\mathrm{CDCl}_{3}\right): \delta=39.4\left(\mathrm{CH}_{2}\right), 40.1\left(\mathrm{CH}_{2}\right), 42.2\left(\mathrm{CH}_{2}\right), 51.8\left(\mathrm{OCH}_{3}\right)$, $55.9\left(\mathrm{OCH}_{3}\right), 56.1\left(\mathrm{OCH}_{3}\right), 112.6(\mathrm{CH}), 115.3(\mathrm{CH}), 126.1(\mathrm{CH}), 128.4$ (2 $\times \mathrm{CH}), 128.5(2 \times \mathrm{Ar}-\mathrm{H}), 128.9(\mathrm{C}), 129.1(\mathrm{C}), 141.3(\mathrm{C}), 147.3(\mathrm{CH})$, $151.6\left(\mathrm{Ar}^{-} \mathrm{OCH}_{3}\right), 172.2(\mathrm{C}=\mathrm{O}), 200.3(\mathrm{C}=\mathrm{O})$.

MS: $m / z(\%)=342(8)\left[\mathrm{M}^{+}\right], 209(100)$.

Anal. Calcd for $\mathrm{C}_{20} \mathrm{H}_{22} \mathrm{O}_{5}: \mathrm{C}, 70.16 ; \mathrm{H}, 6.48$. Found: $\mathrm{C}, 70.45 ; \mathrm{H}, 6.41$.

\section{Methyl 2-(2-(3-(3,4-Dimethoxyphenyl)propanoyl)-4,5-dimethoxy- phenyl)acetate (18d)}

Yield: $89 \mathrm{mg}$ (58\%); colourless oil.

IR (NaCl): $1735(\mathrm{C}=0), 1671(\mathrm{C}=0) \mathrm{cm}^{-1}$.

${ }^{1} \mathrm{H} \mathrm{NMR}\left(\mathrm{CDCl}_{3}\right): \delta=2.97\left(\mathrm{t}, J=7.4 \mathrm{~Hz}, 2 \mathrm{H}, \mathrm{CH}_{2}\right), 3.22(\mathrm{t}, J=7.4 \mathrm{~Hz}$, $\left.2 \mathrm{H}, \mathrm{CH}_{2}\right), 3.71\left(\mathrm{~s}, 3 \mathrm{H}, \mathrm{OCH}_{3}\right), 3.80-3.97\left(\mathrm{~m}, 14 \mathrm{H}, 4 \times \mathrm{OCH}_{3}+\mathrm{CH}_{2}\right)$, 6.70-6.90 (m, 4 H, Ar-H), 7.18-7.31 (m, $1 \mathrm{H}, \mathrm{Ar}-\mathrm{H})$.

${ }^{13} \mathrm{C} \mathrm{NMR}\left(\mathrm{CDCl}_{3}\right): \delta=30.0\left(\mathrm{CH}_{3}\right), 40.0\left(\mathrm{CH}_{2}\right), 42.4\left(\mathrm{CH}_{2}\right), 51.7\left(\mathrm{OCH}_{3}\right)$, $55.7\left(\mathrm{OCH}_{3}\right), 55.7\left(\mathrm{OCH}_{3}\right), 55.8\left(\mathrm{OCH}_{3}\right), 56.0\left(\mathrm{OCH}_{3}\right), 111.1(\mathrm{CH}), 111.7$ $(\mathrm{CH}), 112.6(\mathrm{CH}), 115.2(\mathrm{CH}), 120.0(\mathrm{CH}), 128.9(\mathrm{C}), 129.0(\mathrm{C}), 133.8$ (C), $147.2\left(2 \times \mathrm{Ar}-\mathrm{OCH}_{3}\right), 148.7\left(\mathrm{Ar}-\mathrm{OCH}_{3}\right), 151.5\left(\mathrm{Ar}-\mathrm{OCH}_{3}\right), 172.0$ $(\mathrm{C}=0), 200.3(\mathrm{C}=0)$.

MS: $m / z(\%)=402(63)\left[\mathrm{M}^{+}\right], 209(73), 151(100)$.

Anal. Calcd for $\mathrm{C}_{22} \mathrm{H}_{26} \mathrm{O}_{7}$ : C, 65.66; $\mathrm{H}, 6.51$. Found: $\mathrm{C}, 65.65 ; \mathrm{H}, 6.66$.

\section{2-Benzyl-3-hydroxy-1,4-naphthalene-1,4-diones 20; General Pro- cedure}

$t$-BuOK (257 mg, $2.10 \mathrm{mmol}$ ) was added to stirred solutions of 18a, 18b, 18c and 18d $(0.42 \mathrm{mmol})$ in anhydrous $t-\mathrm{BuOH}(5 \mathrm{~mL})$ at $0{ }^{\circ} \mathrm{C}$. The resulting mixtures were heated at reflux for $1.5 \mathrm{~h}$, then cooled and poured into $10 \%$ aqueous $\mathrm{HCl}(10 \mathrm{~mL})$. The new mixtures were extracted with $\mathrm{CHCl}_{3}(3 \times 15 \mathrm{~mL})$ and the combined organic layers were dried with anhydrous $\mathrm{Na}_{2} \mathrm{SO}_{4}$, and concentrated to dryness under vacuum. The residues were purified by column chromatography (1:3, EtOAc/hexane), to afford compounds 20a, 20b, 20c and 20d, respectively.
2-Benzyl-3-hydroxynaphthalene-1,4-dione (20a)

Yield: 65, mg (57\%); orange solid; $\mathrm{mp} 148-150{ }^{\circ} \mathrm{C}\left(\mathrm{MeOH} / \mathrm{Et}_{2} \mathrm{O}\right)$.

IR (NaCl): $3334(\mathrm{OH}), 1655(\mathrm{C}=0), 1640(\mathrm{C}=0) \mathrm{cm}^{-1}$.

${ }^{1} \mathrm{H} \mathrm{NMR}\left(\mathrm{CDCl}_{3}\right): \delta=3.94\left(\mathrm{~s}, 2 \mathrm{H}, \mathrm{CH}_{2}\right), 7.13-7.28(\mathrm{~m}, 3 \mathrm{H}, \mathrm{Ar}-\mathrm{H}), 7.37-$ 7.41 (m, 2 H, Ar-H), 7.46 (s, 1 H, OH), 7.61-7.16 (m, 2 H, Ar-H), 8.03$8.12(\mathrm{~m}, 2 \mathrm{H}, \mathrm{Ar}-\mathrm{H})$.

${ }^{13} \mathrm{C}$ NMR $\left(\mathrm{CDCl}_{3}\right): \delta=29.1\left(\mathrm{CH}_{2}\right), 123.0(\mathrm{C}), 126.1(\mathrm{CH}), 126.3(\mathrm{CH})$, $126.9(\mathrm{CH}), 128.4(2 \times \mathrm{Ar}-\mathrm{H}), 129.2(2 \times \mathrm{CH}), 129.3(\mathrm{C}), 132.7(\mathrm{C})$, $132.9(\mathrm{CH}), 134.9(\mathrm{Ar}-\mathrm{H}), 138.9(\mathrm{C}), 153.0(\mathrm{CH}), 181.6(\mathrm{C}=\mathrm{O}), 184.3$ $(\mathrm{C}=0)$.

MS: $m / z(\%)=264(100)\left[\mathrm{M}^{+}\right]$.

HRMS (ESI): $m / z$ [M $+\mathrm{H}]^{+}$calcd for $\mathrm{C}_{17} \mathrm{H}_{13} \mathrm{O}_{3}$ : 265.0859; found: 265.0843 .

2-(3,4-Dimethoxybenzyl)-3-hydroxynaphthalene-1,4-dione (20b) Yield: $56 \mathrm{mg}$ (72\%); red solid; $\mathrm{mp} 160-162{ }^{\circ} \mathrm{C}(\mathrm{MeOH})$. IR (NaCl): $3355(\mathrm{OH}), 1659(\mathrm{C}=0), 1638(\mathrm{C}=0) \mathrm{cm}^{-1}$. ${ }^{1} \mathrm{H} \mathrm{NMR}\left(\mathrm{CDCl}_{3}\right): \delta=3.82\left(\mathrm{~s}, 3 \mathrm{H}, \mathrm{OCH}_{3}\right), 3.86\left(\mathrm{~s}, 3 \mathrm{H}, \mathrm{OCH}_{3}\right), 3.88(\mathrm{~s}$, $\left.2 \mathrm{H}, \mathrm{CH}_{2}\right), 6.75(\mathrm{~d}, J=8 \mathrm{~Hz}, 1 \mathrm{H}, \mathrm{Ar}-\mathrm{H}), 6.92-6.96(\mathrm{~m}, 2 \mathrm{H}, \mathrm{Ar}-\mathrm{H}), 7.47$ (s, $1 \mathrm{H}, \mathrm{Ar}-\mathrm{H}), 7.63-7.77$ (m, $2 \mathrm{H}, \mathrm{Ar}-\mathrm{H}), 8.04-8.13$ (m, $2 \mathrm{H}, \mathrm{Ar}-\mathrm{H})$

${ }^{13} \mathrm{C} \mathrm{NMR}\left(\mathrm{CDCl}_{3}\right): \delta=28.6\left(\mathrm{CH}_{2}\right), 55.8\left(2 \times \mathrm{OCH}_{3}\right), 111.8(\mathrm{CH}), 112.6$ $(\mathrm{CH}), 121.1(\mathrm{CH}), 123.2(\mathrm{C}), 126.1(\mathrm{CH}), 126.9(\mathrm{CH}), 129.3(\mathrm{C}), 131.3$ (C), $132.7(\mathrm{C}), 132.9(\mathrm{CH}), 134.9(\mathrm{CH}), 147.5(\mathrm{C}), 148.7(\mathrm{C}), 152.8(\mathrm{C})$, $181.7(\mathrm{C}=0), 184.5(\mathrm{C}=\mathrm{O})$.

MS: $m / z(\%)=324(94)\left[\mathrm{M}^{+}\right], 293$ (100), 138 (53).

HRMS (ESI): $m / z[M+H]^{+}$calcd for $\mathrm{C}_{19} \mathrm{H}_{17} \mathrm{O}_{5}$ : 325.1071; found: 325.1089 .

\section{2-Benzyl-3-hydroxy-6,7-dimethoxynaphthalene-1,4-dione (20c)}

Yield: $63 \mathrm{mg}$ (54\%); red solid; $\mathrm{mp} 148{ }^{\circ} \mathrm{C}$ (decomp).

IR ( $\mathrm{NaCl}): 3341(\mathrm{OH}), 1638(\mathrm{C}=\mathrm{O}) \mathrm{cm}^{-1}$.

${ }^{1} \mathrm{H} \mathrm{NMR}\left(\mathrm{CDCl}_{3}\right): \delta=3.90\left(\mathrm{~s}, 2 \mathrm{H}, \mathrm{CH}_{2}\right), 3.98\left(\mathrm{~s}, 3 \mathrm{H}, \mathrm{OCH}_{3}\right), 4.00(\mathrm{~s}, 3 \mathrm{H}$, OCH3), 7.12-7.31 (m, $4 \mathrm{H}, 4 \times \mathrm{Ar}-\mathrm{H}), 7.35-7.41(\mathrm{~m}, 2 \mathrm{H}, \mathrm{Ar}-\mathrm{H}+\mathrm{OH})$, 7.45 (s, $1 \mathrm{H}, \mathrm{Ar}-\mathrm{H}), 7.54$ (s, $1 \mathrm{H}, \mathrm{Ar}-\mathrm{H})$.

${ }^{13} \mathrm{C} \mathrm{NMR}\left(\mathrm{CDCl}_{3}\right): \delta=29.0\left(\mathrm{CH}_{2}\right), 55.5\left(\mathrm{OCH}_{3}\right), 56.5\left(\mathrm{OCH}_{3}\right), 107.6(\mathrm{CH})$, $108.7(\mathrm{CH}), 121.7(\mathrm{C}), 123.4(\mathrm{C}), 126.2(\mathrm{CH}), 128.0(\mathrm{C}), 128.4(2 \times \mathrm{CH})$, $129.1(2 \times \mathrm{CH}), 139.1(\mathrm{C}), 152.5(\mathrm{C}), 152.8(\mathrm{C}), 154.4(\mathrm{C}), 180.9(\mathrm{C}=\mathrm{O})$, $184.2(\mathrm{C}=\mathrm{O})$.

MS: $m / z(\%)=324(100)\left[\mathrm{M}^{+}\right]$.

HRMS (ESI): $m / z[M+H]^{+}$calcd for $\mathrm{C}_{19} \mathrm{H}_{17} \mathrm{O}_{5}$ : 325.1071; found: 325.1058 .

2-(3,4-Dimethoxybenzyl)-3-hydroxy-6,7-dimethoxynaphthalene1,4-dione (20d)

Yield: $43 \mathrm{mg}$ (39\%); red solid; $\mathrm{mp} 180{ }^{\circ} \mathrm{C}(\mathrm{MeOH})$.

IR ( $\mathrm{NaCl}): 3312(\mathrm{OH}), 1652(\mathrm{C}=0), 1646(\mathrm{C}=\mathrm{O}) \mathrm{cm}^{-1}$.

${ }^{1} \mathrm{H} \mathrm{NMR}\left(\mathrm{CDCl}_{3}\right): \delta=3.82\left(\mathrm{~s}, 3 \mathrm{H}, \mathrm{OCH}_{3}\right), 3.84\left(\mathrm{~s}, 2 \mathrm{H}, \mathrm{CH}_{2}\right), 3.86(\mathrm{~s}, 3 \mathrm{H}$, $\left.\mathrm{OCH}_{3}\right), 3.99\left(\mathrm{~s}, 3 \mathrm{H}, \mathrm{OCH}_{3}\right), 4.01\left(\mathrm{~s}, 3 \mathrm{H}, \mathrm{OCH}_{3}\right), 6.76(\mathrm{~d}, J=8.1 \mathrm{~Hz}, 1 \mathrm{H}$, Ar-H), 6.90-6.98 (m, 2 H, Ar-H), 6.46 (s, 1 H, Ar-H), 7.55 (m, 2 H, Ar$\mathrm{H})$.

${ }^{13} \mathrm{C} \mathrm{NMR}\left(\mathrm{CDCl}_{3}\right): \delta=28.5\left(\mathrm{CH}_{2}\right), 55.8\left(2 \times \mathrm{OCH}_{3}\right), 56.4\left(\mathrm{OCH}_{3}\right), 56.5$ $\left(\mathrm{OCH}_{3}\right), 107.5(\mathrm{CH}), 108.7(\mathrm{CH}), 111.0(\mathrm{CH}), 112.5(\mathrm{CH}), 121.0(\mathrm{CH})$ 121.9 (C), 123.4 (C), 127.9 (C), 132.6 (C), 147.4 (C), 148.7 (C), 152.5 (C), $152.6(\mathrm{C}), 154.4(\mathrm{C}), 180.1(\mathrm{C}=0), 184.2(\mathrm{C}=0)$.

MS: $m / z(\%)=384(55)\left[\mathrm{M}^{+}\right], 353(46), 138(100)$. 
HRMS (ESI): $m / z[M+H]^{+}$calcd for $\mathrm{C}_{21} \mathrm{H}_{21} \mathrm{O}_{7}$ : 385.1282; found: 385.1276.

\section{Funding Information}

This work has received financial support from the Xunta de Galicia (Centro singular de investigación de Galicia accreditation 2016-2019, ED431G/09; and Project GRC2014/040), the European Union (European Regional Development Fund-ERDF), FONDECYT (Research Grants 1161816 and 1141264) and Galchimia S.A.

\section{Supporting Information}

Supporting information for this article is available online at https://doi.org/10.1055/s-0036-1591729.

\section{References}

(1) Tandon, V. K.; Kumar, S. Expert Opin. Ther. Pat. 2013, 23, 1087.

(2) Klotz, L. O.; Hou, X.; Jacob, C. Molecules 2014, 19, 14902.

(3) Salas, C. O.; Faundez, M.; Morello, A.; Maya, J. D.; Tapia, R. A. Curr. Med. Chem. 2011, 18, 144.

(4) Kumagai, Y.; Shinkai, Y.; Miura, T.; Cho, A. K. Annu. Rev. Pharmacol. Toxicol. 2012, 52, 221.

(5) Ogata, T.; Yoshida, T.; Shimizu, M.; Tanaka, M.; Fukuhara, C.; Ishii, J.; Nishiuchi, A.; Inamoto, K.; Kimachi, T. Tetrahedron 2016, $72,1423$.

(6) Fiorito, S.; Epifano, F.; Bruyère, C.; Mathieu, V.; Kiss, R.; Genovese, S. S. Bioorg. Med. Chem. Lett. 2014, 24, 454.

(7) Lu, X.; Altharawi, A.; Gut, J.; Rosenthal, P. J.; Long, T. E. ACS Med. Chem. Lett. 2012, 3, 1029.

(8) Shaabani, S.; Naimi-Jamal, R. M.; Ali, Maleki. A. Dyes Pigm. 2015, $122,46$.

(9) Salas, C. O.; Reboredo, F. J.; Estévez, J. C.; Tapia, R. A.; Estévez, R. J. Synlett 2009, 3107.

(10) Cruces, J.; Martinez, E.; Treus, M.; Martinez, L. A.; Estévez, J. C.; Estévez, R. J.; Castedo, L. Tetrahedron 2002, 58, 3015.

(11) Martinez, A.; Fernández, M.; Estévez, J. C.; Estévez, R. J.; Castedo, L. Tetrahedron 2005, 61, 1353.

(12) Lee, Y. R.; Kim, B. S. Synth. Commun. 2003, 33, 4123.

(13) Qabaja, G.; Perchellet, E. M.; Perchellet, J.-P.; Jones, G. B. Tetrahedron Lett. 2000, 41, 3007.

(14) Qabaja, G.; Jones, G. B. J. Org. Chem. 2000, 65, 7187.

(15) Poljakova, J.; Eckschlager, T.; Hrabeta, J.; Hrebackova, J.; Smutny, S.; Frei, E.; Martinek, V.; Kizek, R.; Stiborova, M. Biochem. Pharmacol. 2009, 77, 1466.

(16) Moody, D. L.; Dyba, M.; Kosakowska-Cholody, T.; Tarasova, N. I.; Michejda, C. J. Bioorg. Med. Chem. Lett. 2007, 17, 2380.

(17) Louvis, A. R.; Silva, N. A. A.; Semaan, F. S.; Silva, F. C.; Saramago, G.; Souza, L. C. S. V.; Ferreira, B. L. A.; Castro, H. C.; Salles, J. P.; Souza, A. L. A.; Faria, R. X.; Ferreira, V. F.; Martins, D. L. New J. Chem. 2016, 40, 7643.

(18) Svoboda, G. H.; Poore, G. A.; Montfort, M. L. J. Pharm. Sci. 1968, $57,1720$.

(19) Sehgal, S. N.; Czerkawski, H.; Kudelski, A.; Pandev, K.; Saucier, R.; Vezina, C. J. Antibiot. 1983, 36, 355

(20) Findlay, J. A.; Liu, J. S.; Radics, L.; Rakhit, S. Can. J. Chem. 1981, 59, 3018.
(21) Findlay, J. A.; Liu, J. S.; Radics, L. Can. J. Chem. 1983, 61, 323.

(22) Cheng, C. C.; Ellie, G. P.; West, G. B. Structural Aspects of Antineoplastic Agents-A New Approach; Elsevier: Amsterdam, 1988, 25 p. 35-83.

(23) Chang, H. X.; Chou, T. C.; Savaraj, N.; Liu, L. F.; Yu, C.; Cheng, C. C. J. Med. Chem. 1999, 42, 405.

(24) Rhee, H.-K.; Kwon, Y.; Chung, H.-J.; Lee, S. K.; Choo, H.-Y. P. Bull. Korean Chem. Soc. 2011, 32, 2391.

(25) Asche, C.; Frank, W.; Albert, A.; Kucklaender, U. Bioorg. Med. Chem. 2005, 13, 819.

(26) Cruces, J.; Estévez, J. C.; Castedo, L.; Estévez, R. J. Tetrahedron Lett. 2001, 42, 4825.

(27) Barcia, J. C.; Cruces, J.; Estévez, J. C.; Estévez, R. J.; Castedo, L. Tetrahedron Lett. 2002, 43, 5141.

(28) Buu-Hoi, N. P.; Jacquignon, P.; Mangane, M. Recl. Trav. Chim. Pays-Bas 1965, 84, 334.

(29) Liu, K.; Wood, H. B.; Jones, A. B. Tetrahedron Lett. 1999, 40, 5119.

(30) Valderrama, J. A.; Espinoza, O.; González, M. F.; Tapia, R. A.; Rodríguez, J. A.; Theodulozb, C.; Schmeda-Hirschmannb, G. Tetrahedron 2006, 62, 2631.

(31) Martinez, E.; Martinez, L.; Treus, M.; Estévez, J. C.; Estévez, R. J.; Castedo, L. Tetrahedron 2000, 56, 6023.

(32) Stagliano, K. W.; Malinakova, H. C. J. Org. Chem. 1999, 64, 8034

(33) Cabri, W.; Candiani, I.; Bedeschi, A.; Penco, S.; Santi, R. J. Org. Chem. 1992, 57, 1481.

(34) Overman, L. E.; Poon, D. J. Angew. Chem. Int. Ed. Engl. 1997, 36, 518.

(35) Ruan, J. W.; Xiao, J. L. Acc. Chem. Res. 2011, 44, 614.

(36) Ruan, J.; Iggo, J. A.; Berry, N. G.; Xiao, J. J. Am. Chem. Soc. 2010, $132,16689$.

(37) Svennebring, A.; Sjoeberg, P. J. R.; Larhed, M.; Nilsson, P. Tetrahedron 2008, 64, 1808.

(38) Cabri, W.; Candiani, I. Acc. Chem. Res. 1995, 28, 2.

(39) Yang, R.-Y.; Kizer, D.; Wu, H.; Volckova, E.; Miao, X.-S.; Ali, S. M.; Tandon, M.; Savage, R. E.; Chan, T. C. K.; Ashwell, M. A. Bioorg. Med. Chem. 2008, 16, 5635.

(40) Martinez, A.; Estévez, J. C.; Estévez, R. J.; Castedo, L. Tetrahedron Lett. 2000, 41, 2365.

(41) Huanli, X.; Qunying, C.; Hong, W.; Pingxiang, X.; Ru, Y.; Xiaorong, L.; Lu, B.; Ming, X. J. Exp. Clin. Cancer Res. 2016, 35, 178.

(42) Fiorito, S.; Epifano, F.; Bruyere, C.; Mathieu, V.; Kiss, R.; Genovese, S. Bioorg. Med. Chem. Lett. 2014, 24, 454.

(43) Lu, J.-J.; Bao, J.-L.; Wu, G.-S.; Xu, W.-S.; Huang, M.-Q.; Chen, X.P.; Wang, Y.-T. Anti-Cancer Agents Med. Chem. 2013, 13, 456.

(44) Salas, C.; Tapia, R. A.; Ciudad, K.; Armstrong, V.; Orellana, M.; Kemmerling, U.; Ferreira, J.; Maya, J. D.; Morello, A. Bioorg. Med. Chem. 2008, 16, 668.

(45) Ogata, T.; Yoshida, T.; Shimizu, M.; Tanaka, M.; Fukuhara, C.; Ishii, J.; Nishiuchi, A.; Inamoto, K.; Kimachi, T. Tetrahedron 2016, $72,1423$.

(46) Ferreira, S. B.; Rodrigues da Rocha, D.; Carneiro, J. W. M.; Santos, W. C.; Ferreira, V. F. Synlett 2011, 1551.

(47) Ramachary, D. B.; Kishor, M. Org. Biomol. Chem. 2010, 8, 2859.

(48) Hooker, S. C. J. Org. Chem. 1936, 58, 1174.

(49) Roushdi, I. M.; Mikhail, A. A.; Chaaban, I. Pharmazie 1976, 31, 406.

(50) Kobayashi, K.; Matsunaga, A.; Mano, M.; Morikawa, O.; Konishi, H. Heterocycles 2002, 57, 1915. 